Article

\title{
Reduction of Uncorrelated Striping Noise-Applications for Hyperspectral Pushbroom Acquisitions
}

\author{
Christian Rogass ${ }^{1, *}$, Christian Mielke ${ }^{1}$, Daniel Scheffler ${ }^{1}$, Nina K. Boesche ${ }^{1}$, Angela Lausch ${ }^{2}$, \\ Christin Lubitz ${ }^{1}$, Maximilian Brell ${ }^{1}$, Daniel Spengler ${ }^{1}$, Andreas Eisele ${ }^{1}$, Karl Segl ${ }^{1}$ \\ and Luis Guanter ${ }^{1}$
}

1 Helmholtz Center Potsdam, German Research Center for Geosciences, Telegrafenberg, Potsdam 14473, Germany; E-Mails: christian.mielke@gfz-potsdam.de (C.M.); daniel.scheffler@gfz-potsdam.de (D.S); nina.boesche@gfz-potsdam.de (N.K.B.); christin.lubitz@gfz-potsdam.de (C.L.); maximilian.brell@gfz-potsdam.de (M.B.); daniel.spengler@gfz-potsdam.de (D.S.); andreas.eisele@gfz-potsdam.de (A.E.); karl.segl@gfz-potsdam.de (K.S.); luis.guanter@gfz-potsdam.de (L.G.)

2 Helmholtz Center for Environmental Research-UFZ, Permoserstr 15, Leipzig 04318, Germany; E-Mail: angela.lausch@ufz.de

* Author to whom correspondence should be addressed; E-Mail: christian.rogass@gfz-potsdam.de; Tel.: +49-331-288-1820; Fax: +49-331-288-1192.

External Editors: Richard Gloaguen and Prasad S. Thenkabail

Received: 5 September 2014; in revised form: 30 October 2014 / Accepted: 4 November 2014 / Published: 11 November 2014

\begin{abstract}
Hyperspectral images are of increasing importance in remote sensing applications. Imaging spectrometers provide semi-continuous spectra that can be used for physics based surface cover material identification and quantification. Preceding radiometric calibrations serve as a basis for the transformation of measured signals into physics based units such as radiance. Pushbroom sensors collect incident radiation by at least one detector array utilizing the photoelectric effect. Temporal variations of the detector characteristics that differ with foregoing radiometric calibration cause visually perceptible along-track stripes in the at-sensor radiance data that aggravate succeeding image-based analyses. Especially, variations of the thermally induced dark current dominate and have to be reduced. In this work, a new approach is presented that efficiently reduces dark current related stripe noise. It integrates an across-effect gradient minimization principle. The performance has been evaluated using artificially degraded whiskbroom (reference) and real pushbroom
\end{abstract}


acquisitions from EO-1 Hyperion and AISA DUAL that are significantly covered by stripe noise. A set of quality indicators has been used for the accuracy assessment. They clearly show that the new approach outperforms a limited set of tested state-of-the-art approaches and achieves a very high accuracy related to ground-truth for selected tests. It may substitute recent algorithms in the Reduction of Miscalibration Effects (ROME) framework that is broadly used to reduce radiometric miscalibrations of pushbroom data takes.

Keywords: stripes; hyperspectral; dark current; pushbroom; calibration; radiometric; EO-1 Hyperion; AISA; EnMAP; ROME

\section{Introduction}

Remote sensing data acquisitions broadly serve as a basis for spatiotemporal analyses of the status and the dynamic of the Earth's surface. The increasing demand for spatially highly resolved geoinformation rises the quantity of different sensors and acquisition platforms. Optical systems such as imaging spectrometers offer continuous spectra on a pixel basis enabling the identification and quantification of surface cover materials due to their spectral response on incident electromagnetic radiation. Imaging spectrometers that follow the line scanner principle utilize either the whiskbroom technology such as the airborne HyMAP (Hyperspectral Mapper) [1] or the pushbroom technology such as the spaceborne EO-1 Hyperion [2] or the airborne AISA (Airborne Imaging Spectrometer for Applications) Dual [3]. Most of them provide continuous spectra that ranges from the visible and near infrared (VNIR: $0.4-1.3 \mu \mathrm{m}$ ) up to the short wave infrared (SWIR: $1.3-2.5 \mu \mathrm{m}$ ) wavelength region to support a broad set of applications. Scanners that utilize the pushbroom technology are mostly used because a longer integration time enables a better Signal-To-Noise Ratio (SNR) compared to whisk-broom scanners. Each pixel of a pushbroom acquisition represents the data take of one detector column of the detector array or briefly a spectrum. If the physical characteristics of detector elements vary over time and, hence, differ from the current calibration set, then stripes are visually perceptible after the radiometric scaling with the current calibration set. With regard to [4-6], such effects can be considered as miscalibration and may consist of linear and non-linear signal dependent and independent fractions. The independent fractions are caused by variations of thermally induced electron pairs that are broadly considered as dark current or offset. The thermally induced dark current variations are Gaussian distributed (Gaussian White Noise) and signal independent as described in [5,7,8]. In [4,9], approaches have been proposed that aim to reduce miscalibration effects. The framework has been named ROME - Reduction of Miscalibration Effects - and is currently used by different airborne facilities to correct the acquisitions for miscalibrations. However, proposed approaches of [4,9] lack a high precision for the dark current estimation. Thus, a new approach has been developed that is analytically derived and contemporarily achieves a significant higher accuracy than the approaches proposed in [4,9-14] that partly belong to the reviewed approaches of [15]. There are other techniques published in the literature [16-36] as stated in $[4,15]$, but they were not tested in this paper and may be the focus of future reviewing work, which should also comprise more performance benchmarks to test the capacities of the approaches [16-36] to be integrated into ROME or other radiometry related frameworks. 
For validation, a large set of artificially degraded and real hyperspectral image samples from both airborne and spaceborne acquisitions has been utilized, which is described in more detail in the following section. In the next sections, an approach is presented that integrates human visual perception of miscalibration related across-track gradients by minimizing their modulus.

\section{Materials and Methods}

\subsection{Materials}

Real hyperspectral images and artificially degraded samples were used to evaluate the performance of inspected approaches. The samples are comprised of three atmospherically corrected airborne HyMAP [1] scenes, two radiometrically corrected spaceborne EO-1 Hyperion [2] scenes and two radiometrically corrected airborne AISA Dual [3] scenes. The HyMAP scenes were acquired over Germany in detail; over Potsdam in 2004, over Berlin in 2005 and over Dresden in 2003 with an average ground sampling distance (GSD) of about $6 \mathrm{~m}$. The Hyperion scenes were acquired in 2013 and 2014 over the Haib River Complex and cross the border region of Namibia and the Republic of South Africa with an average ground sampling distance of about $30 \mathrm{~m}$. The AISA Dual scenes were acquired over the Fichtwald region, Germany, in 2010 with an average ground sampling distance of about $2 \mathrm{~m}$. The locations of the case study regions are shown in Figure 1.

Figure 1. Overview on study regions as Landsat 8 false color composite subsets overlaid with vectorized acquisition borders for airborne data takes (a)-two scenes over the Fichtwald region (AISA DUAL; red), spaceborne data takes (b)-two scenes over the Haib River Complex of Namibia and the Republic of South Africa (Hyperion; green) and airborne data takes (c,d) three scenes over the cities of Dresden (c), Berlin, Potsdam (HyMap; blue).

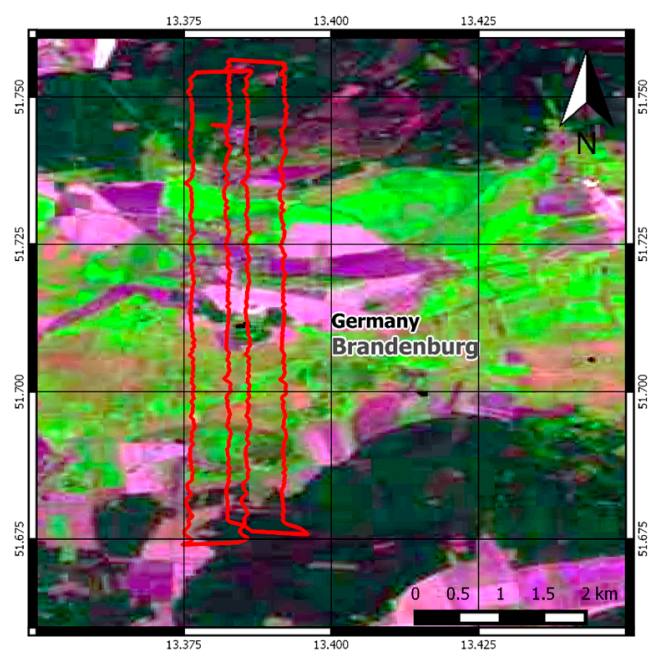

(a)

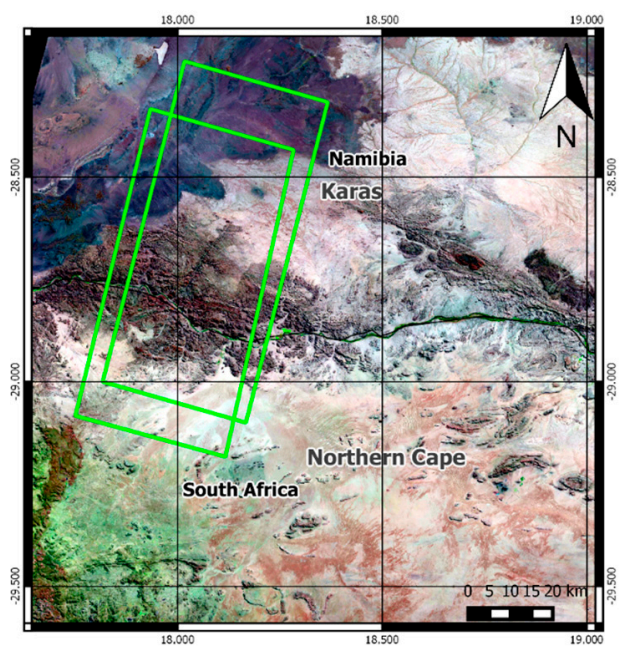

(b) 
Figure 1. Cont.

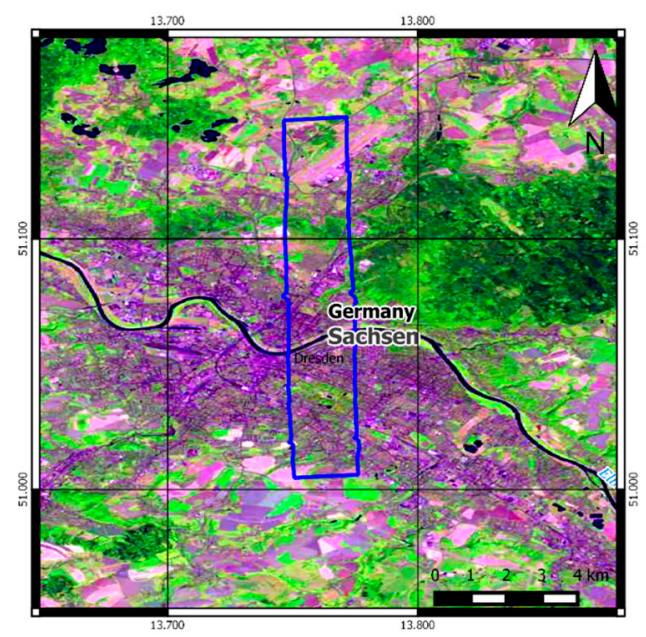

(c)

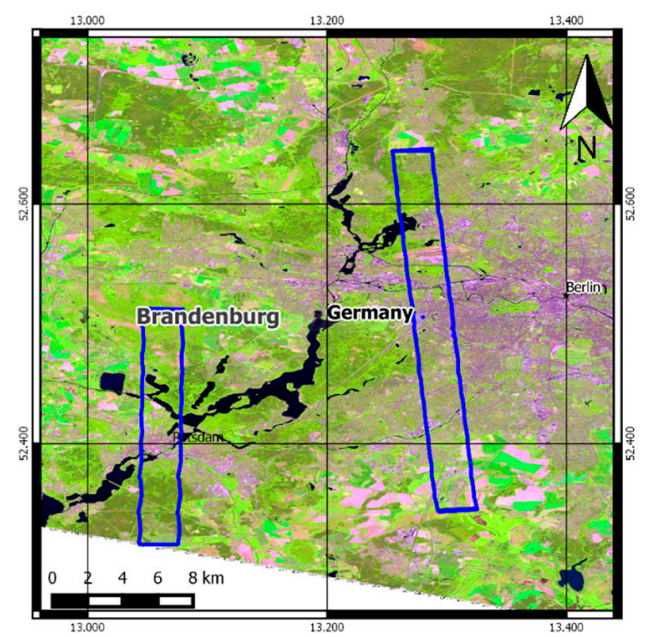

(d)

The HyMAP [1] system is primarily used for hyperspectral data taken from airborne platforms. It utilizes the whiskbroom technology (rotating mirror) and has about 128 spectral bands (depending on configuration). HyMAP continuously covers the spectral region between 0.44 and $2.5 \mu \mathrm{m}$ and provides spectral resolution from 15 to $20 \mathrm{~nm}$. Contrary to pushbroom systems, data acquisitions of single detector systems such as those following the whisk-broom scanning principle are assumed to remain radiometrically stable in across-track direction and, hence, are assumed to be not impacted by temporal dark current variations. Therefore, they have been selected as the optimal basis of comparison for simulations to evaluate the performance of different destriping approaches for pushbroom systems such as AISA, Hyperion, or in the future EnMAP [37,38]. The HyMAP scenes have been atmospherically corrected through ATCOR [39] before artificial degradation has been applied. Additionally, all bands of the scenes have been visually inspected to identify potential remaining stripes that have to be corrected before artificial degradation. No HyMAP scenes were impacted by striping noise, as it is likely for whiskbroom acquisitions as proposed in [1]. However, atmospheric correction has been performed before artificial degradation to avoid drawbacks from varying illumination geometry on the evaluation of the performances of tested destriping approaches. Remaining long waves across track gradients have been reduced using the detrending approach of [4]. This was essential for an objective performance evaluation of the artificial degraded HyMAP images, although the destriping should always be applied before atmospheric correction using at-sensor radiance or at-sensor reflectance. Subsets of these scenes are exemplarily shown in Figure 2a,c.

The hyperspectral Hyperion system is mounted on the spaceborne EO-1 platform [2], has about 220 bands, acquires data from a sun-synchronous 705-km-high orbit with an average ground sampling distance of about $30 \mathrm{~m}$ and continuously covers a spectral region between 0.4 and $2.5 \mu \mathrm{m}$. Contrary to HyMAP or AISA, some bands of Hyperion are erroneous or are spectrally redundant [40] and, hence, those bands have not been considered, which resulted in 196 bands of 220 and ranging from $0.426 \mu \mathrm{m}$ to $2.395 \mu \mathrm{m}$. Additionally, the Hyperion at-sensor-radiance (L1R product) scenes have been corrected for missing data as proposed in [10]. Subsets of these scenes are exemplarily shown in Figure 2d,e. 
The AISA DUAL [3] system is primarily used for hyperspectral data taken from airborne platforms. This system has about 491 bands (depending on configuration) and consists of two sensors utilizing the pushbroom technology (Eagle and Hawk). The Eagle covers the spectral region between 0.4 and $0.97 \mu \mathrm{m}$ (VNIR) with a spectral resolution of about $2.3 \mathrm{~nm}$. The Hawk covers the spectral region between 0.97 and $2.45 \mu \mathrm{m}$ (SWIR) with a spectral resolution of about $6.3 \mathrm{~nm}$ (depending on configuration). Therefore, both AISA systems continuously cover a similar spectral region as HyMAP.

The AISA scenes have been radiometrically corrected using the AISA processing software CaliGeo [3]. Subsets of these scenes are exemplarily shown in Figure 2f,g whereas band combinations for real hyperspectral images were selected that enable a better visual perception of the stripe noise.

Figure 2. False colored representations of subsets of HyMAP acquisitions over (a) Potsdam (CIR: Red $864.5 \mathrm{~nm}$, Green $652.6 \mathrm{~nm}$, Blue $546.3 \mathrm{~nm}$ ), (b) Berlin (CIR as in (a), (c) Dresden and of subsets of Hyperion acquisitions over (d,e) the Haib River Complex (Red $2304.71 \mathrm{~nm}$, Green $915.23 \mathrm{~nm}$, Blue $447.17 \mathrm{~nm}$ ), and subsets of AISA acquisitions over the Fichtwald region (f) and (g) (Red $1574.37 \mathrm{~nm}$, Green $964.39 \mathrm{~nm}$, Blue $730.05 \mathrm{~nm})$.

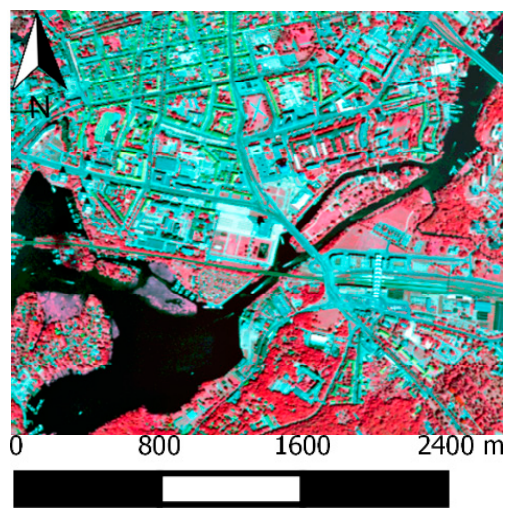

(a)

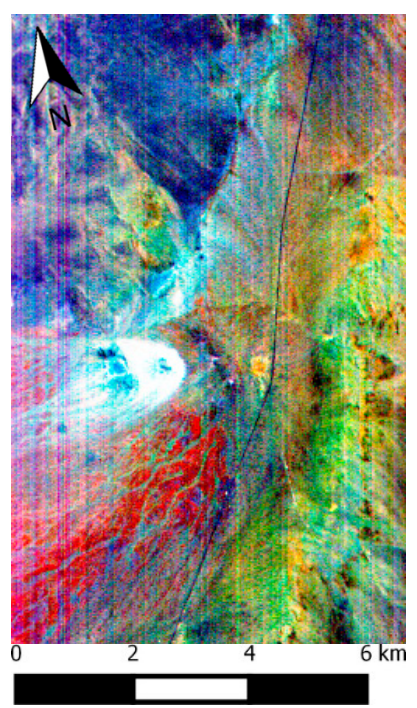

(d)

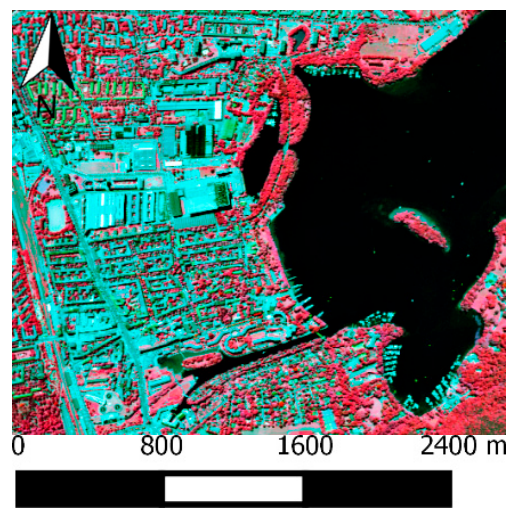

(b)

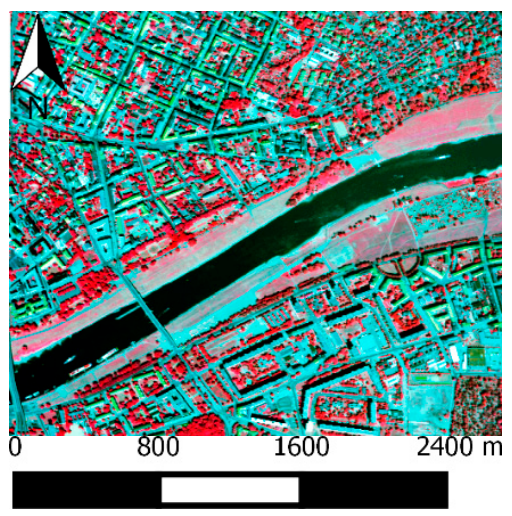

(c)

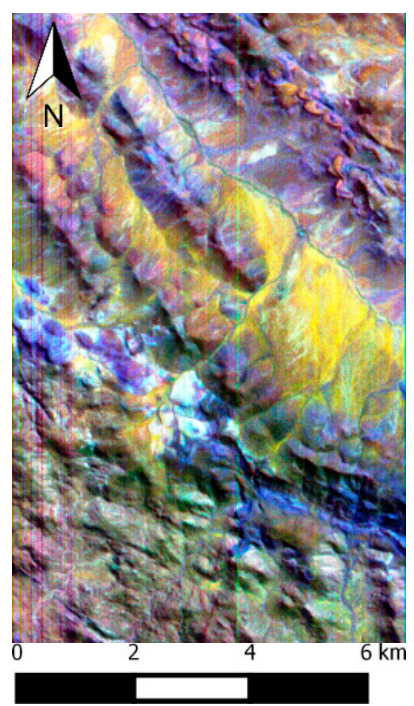

(e)

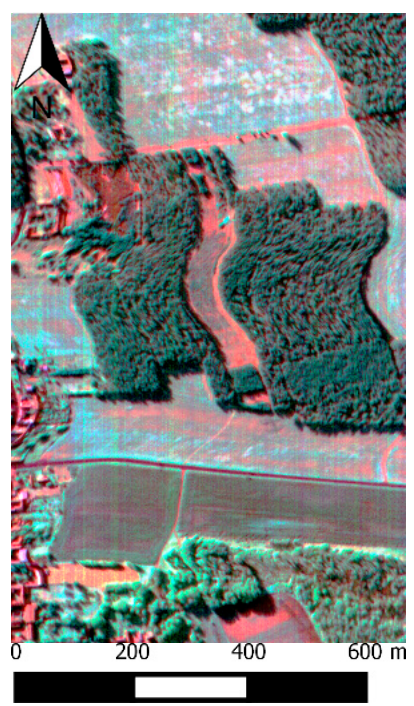

(f)

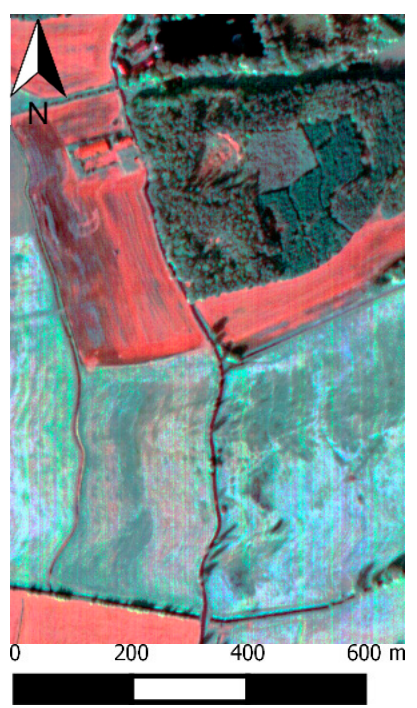

$(\mathbf{g})$

After pre-processing the HyMAP reflectance scenes (Figure 2a,c) were artificially striped across track for succeeding destriping and its related evaluation of tested destriping approaches. This was individually performed for each band of each scene of each scenario by adding White Gaussian Noise (WGN) to the 
bands as proposed in [41], having a zero mean and specific standard deviations, whereas the striping pattern was assumed to be invariant per scene or within a short time span as assumed in $[5,7,8]$. For this, the simulated noise vector was mean normalized and then divided by its standard deviation to have zero mean and a standard deviation of 1 . Then, the noise vector was rescaled to a specific scale (given in the following) and replicated as a row vector to the band dimensions by using the dyadic product between the row noise vector and a column vector valued 1 and of width equal to the number of columns of one band. In this work, WGN was selected for the artificial image degradation, because it has been assumed that dark current variations are caused by variations of thermally induced electrons which probability distribution is a Gaussian according to [5,7,8]. Additionally, it is assumed that the dark current varies only slowly over time and the sensor manufacturer integrated steering elements to keep the detector elements by progressive cooling in thermal balance to provide a nearly scene invariant (slowly varying) dark current. Although those assumptions appear to be valid for testing the performance of different approaches the noise models vary from sensor to sensor as described in [6] that aggravates any method evaluation. However, the different standard deviations represent scaled degradations of four different striping scenarios, whereas scales represent standard deviations of $0.1 \%, 0.5 \%, 1 \%$ and $5 \%$ of the full gray value range (difference of the maximum and the minimum gray value of the band) of the band that shall be degraded. This is exemplarily shown in Figure 3. Hence, 1536 different striping scenarios (4 noise levels, 3 HyMAP images, 128 bands) were simulated to serve as input for the evaluation of inspected destriping approaches, whereas each striping scenario was realized by simple addition to account for the property of dark current to be uncorrelated for the signal.

Figure 3. Examples of false colored (CIR: Red $864.5 \mathrm{~nm}$, Green $652.6 \mathrm{~nm}$, Blue $546.3 \mathrm{~nm}$ ) artificially degraded subsets of HyMAP acquisitions over Potsdam (a-d), over Berlin (e-h) and Dresden (i-l); noise degradation levels (a,e,i) $0.1 \%$, (b,f,j) $0.5 \%$, (c,g,b) $1 \%$ and (d,h,l) 5\% noise degradation.

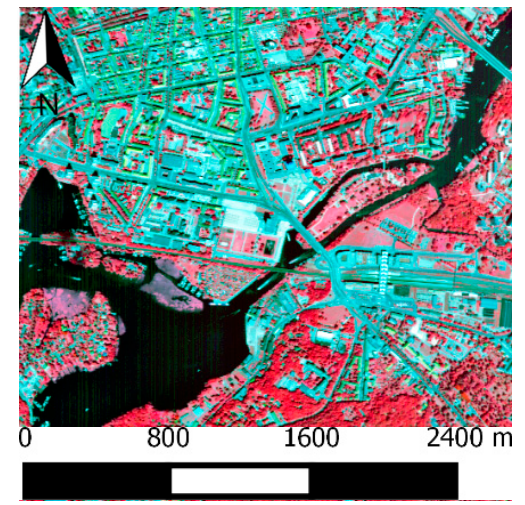

(a)

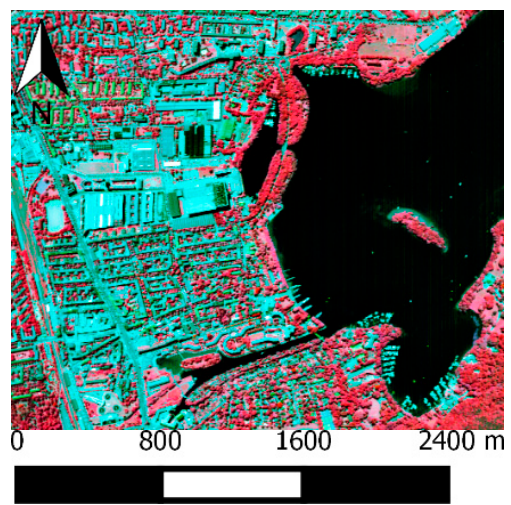

(e)

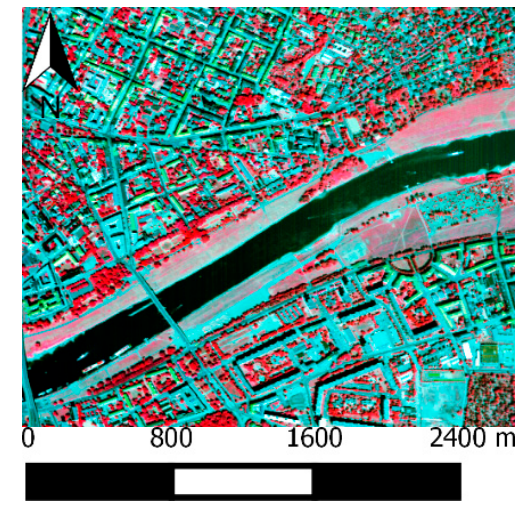

(i) 
Figure 3. Cont.

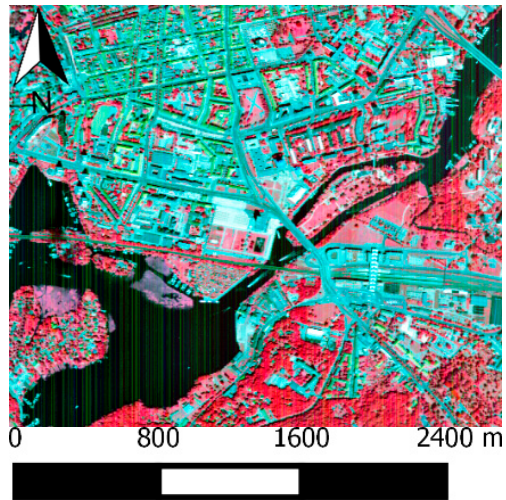

(b)

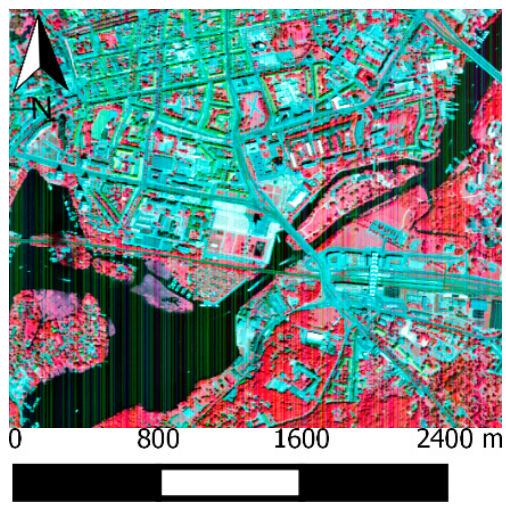

(c)

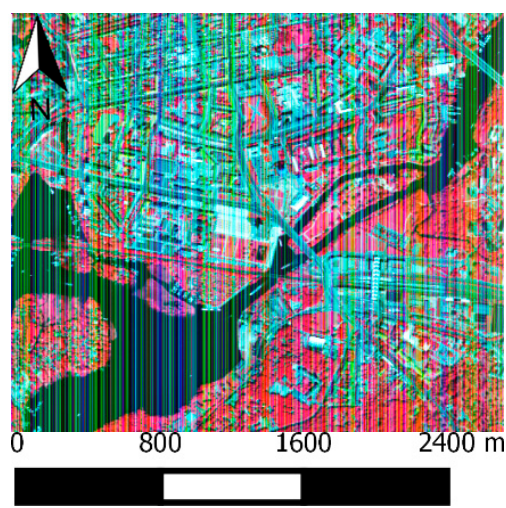

(d)

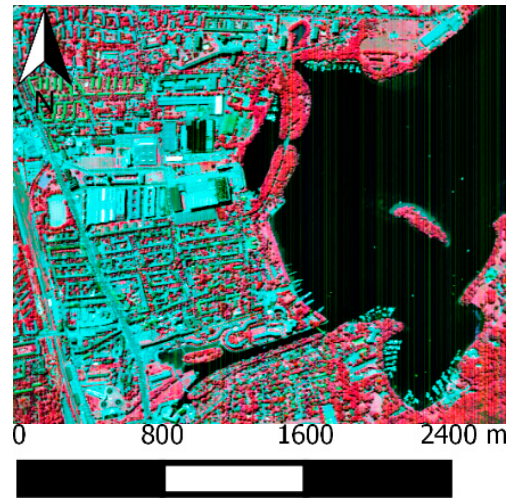

(f)

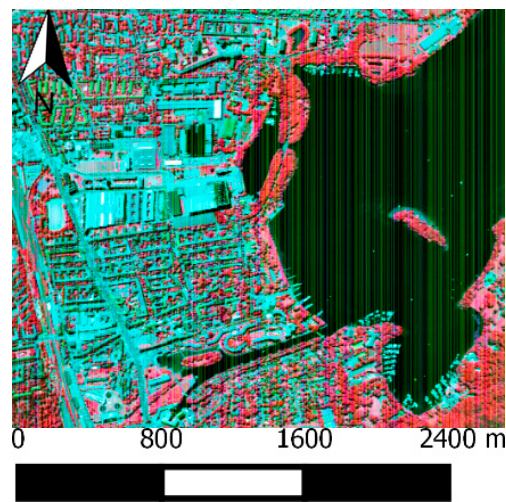

(g)

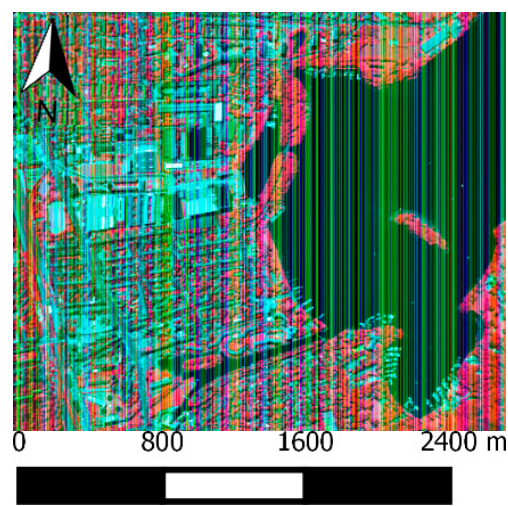

(h)

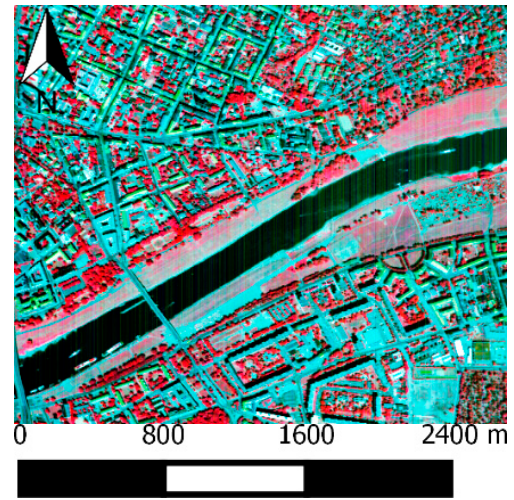

(j)

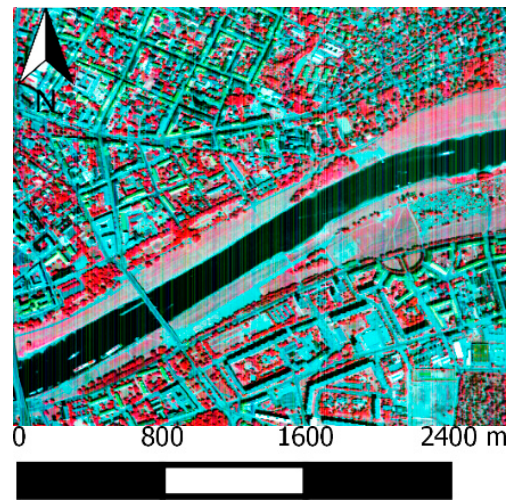

(k)

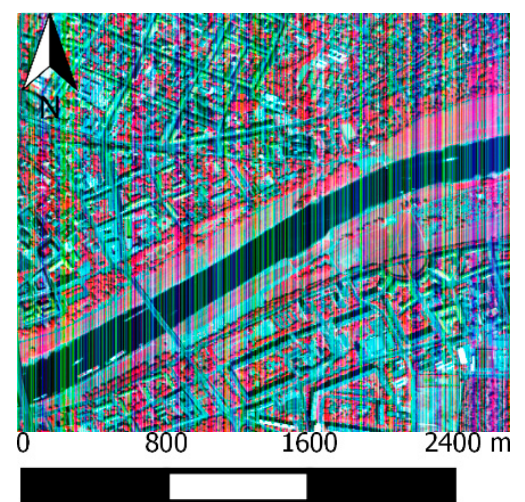

(I)

\subsection{Methods}

\subsubsection{Selected Current Approaches}

Temporal dark current variations lead to uncorrelated striping caused by temporal variations of thermally induced electrons $[5,7,8]$. The signal superimpositions are signal uncorrelated and vary from sensor to sensor. As stated in [4] airborne acquisitions are more affected than spaceborne acquisitions that may be caused by higher dynamics of acquisition conditions. The following convention has been made for succeeding algorithm descriptions and is related to the pushbroom technology and its detector arrays. A pixel is the acquisition of a single detector element, a spectrum is the acquisition of all detector elements of a single detector array column representing all bands, a column or samples are all 
acquisitions of a single detector element (pixel) over time and a row or a line are all detector element (pixels) acquisitions of one band for one integration time step in across-track direction.

In this work, a new approach is proposed and its performance is compared with other broadly used approaches. Those are the approaches proposed in [4,9,11-14] and tested in [15], which will be described shortly in the following section. The approaches of [15] have partly been selected for performance evaluation to be comparable with the results provided by [15]. Related reviewing work should extend the number of tested approaches to provide a better overview on the performance of existing approaches such as those proposed in [16-36], which were not the focus of this work.

In $[4,9]$ new techniques have been proposed that aim to reduce radiometric miscalibration comprising reductions of dark current variation related offset stripes and deviations of radiometric detector linearities as well as non-linearities. The approach of [4] is called ROME-Reduction Of Radiometric Effects. This approach has been tested again in [9], analyzing real images.

In [4,9], at first, column differences are computed. Then, the distribution of all differences of each column are examined due to their likelihood using an approximation of their respective histogram. The histogram bin (frequency category) with the highest likelihood then represents the agglomerated majority of column differences of the inspected column. To avoid drawbacks in relying on a single criterion, all bins are median averaged. All averages are then median averaged, whereby each bin average is weighted by its number of elements. Those weighted averages are then subtracted from the striped images. Afterwards, the destriped image is rescaled using the gray value maximum and minimum before and after destriping. This is performed to preserve the gray value range. In the last step, long wave brightness gradients or reduction undershoots are reduced. For this, the destriped image is divided by the dyadic product of a detector related index vector and a boxcar smoothed vector of median column averages.

In [11] an approach is presented that comprises neither local or global reductions, but both are proceeded using the same scheme. In the following, the global approach is briefly described that has been also used for testing. First, local reductions are performed to remove outliers. Then, global reduction is performed to suppress striping noise.

The general scheme is straightforward and assumes that local or global statistics are not affected by uncorrelated noise. In the global approach, the global standard deviation of this band and the column standard deviations for each pixel are computed. The ratios between the global standard deviation and the local standard deviations are then considered as updated gains alike the gain coefficient that has been estimated in the process of radiometric calibration. The offsets are then estimated as the difference of a global band mean and the product of the column gains and the column means. Both the new offset and the new gain are then applied as in the process of radiometric scaling. The gray values of dead or erroneous detector elements have been corrected beforehand by applying the approach of [10] and the local or the global method can be applied.

In [12], an approach is proposed that is part of an EO-1 Hyperion related processing scheme for at-ground-reflectance retrieval. At first, the column means of each band are computed. Then, a smoothed representation is computed by using triangular smoothing of size 10 . The ratio between the pixel corresponding to the value of the smoothed column mean and the column mean is then applied as new gain. This approach is straightforward, but does not correct for erroneous gains as assumed. It corrects for dark current related miscalibrations as shown in [4], because otherwise the at-sensor-radiance data has to be rescaled back to the digital number (DN) successively reduced with the offsets that have been 
estimated in the process of radiometric miscalibration. However, this approach is quite similar to the inversed equation of the detrending part of the approach of [4].

In [13], an approach is presented that combines wavelet decompositions and frequency domain adaptive filtering per band. First, the image is wavelet decomposed. Then, the vertical components are Fourier transformed into the frequency domain, where basically striping is suppressed using an adaptive iterative filter scheme that aims on differentiating between image background and uncorrelated stripe noise. Afterwards, the Fourier image is transformed back into the wavelet domain and then further back to the spatial domain. Basically, this approach assumes that stripe noise is to some extent stationary.

In [14], an approach is presented that bases on filtering the outputs of the Maximum Noise Fraction Transformation (MNF) of [42] and transforming the results back to the original image domain. The noise covariance matrix of the image that is necessary to perform the MNF is here directly estimated from the image itself by integrating computed statistics of shift differenced homogeneous regions. After MNF transformation, the striped MNF bands are destriped using the approach proposed in [13] and transformed back to the image domain. This approach assumes that stripe noise tends to remain uncorrelated after MNF transformation and is contemporarily conditioned by the implementation of the approach of [13]. It is also assumed that stripe noise is to some extent stationary.

The approaches of [13] and [14] are parameterized and, hence, can be adjusted from image to image to provide better destriping results. In this work the parameters have been used that have been commonly suggested by [13-15].

\subsubsection{Proposed Approach}

The following proposed approach can be considered as an enhancement of the approaches of $[4,9]$ for dark current variation related destriping and updates the major framework of ROME as proposed in $[4,9]$. The basic radiometric rescaling equation that integrates radiometric calibration coefficients is the following that follows the notation of [24]:

$$
L 1(x, y, \lambda)=\frac{D N(x, y, \lambda)-\left(D N_{0}(x, \lambda)+\varepsilon(x, \lambda)\right) \otimes 1_{w}(y, \lambda)}{g(x, \lambda) \otimes 1_{w}(y, \lambda)}
$$

where $L 1(x, y, \lambda)$ denotes at-sensor-radiance, $D N(x, y, \lambda)$ the recorded digital number $(D N), D N_{0}(x, \lambda)$ the dark current estimated in the process of radiometric calibration, $\varepsilon(x, \lambda)$ a scene constant variation of uncorrelated striping noise and $g(x, \lambda)$ the gain respectively, $x$ the detector element or across track position, $y$ the scan line number or along track scan line position, $\lambda$ the wavelength, $1_{w}$ a row vector of width equal to the number of rows and valued 1 and $\otimes$ is the dyadic product. Usually, $\varepsilon(x, \lambda)$ is very small and can be considered as an update between two succeeding dark current estimations. Temporal variations of dark current and path radiance related stray light can potentially cause deviations from foregoing calibration. Hence, striping noise is visually perceptible especially over sensed low albedo regions, where the ratio between the striping noise and the ground reflected radiation is higher than in high albedo regions. Considering only one band and using a shorter notation Equation (1) can be simplified to the following equation: 


$$
\begin{gathered}
L 1=\frac{D N-\left(D N_{0}(x)+\varepsilon(x)\right) \otimes 1_{w}(y)}{g(x) \otimes 1_{w}(y)}=i m=i m_{s}-s \\
s=\frac{\varepsilon(x) \otimes 1_{w}(y)}{g(x) \otimes 1_{w}(y)}
\end{gathered}
$$

where $\mathrm{im}$ denotes the at-sensor radiance image of one band where all uncorrelated striping noise have been completely removed, $\mathrm{im}_{s}$ the real at-sensor radiance image of one band and $s$ the uncorrelated striping noise matrix of one band that represents the uncorrelated, stationary dark current variation or offset miscalibration [5]. Considering $s$ as a signal superimposition of $\mathrm{im}_{s}$, it can be assumed that uncorrelated along track striping noise causes additional across track gradients. Therefore, the basic assumption of this work is that remaining stripes cause additional gradients perpendicular to the stripe direction. This can be expressed for along track stripes by the following integrals for the image defined in the domain $\Omega$ that is a bounded domain of $\Re^{2}$ :

$$
\int_{\Omega} \frac{\partial i m-i m_{s}}{\partial x} d y=\int_{\Omega} \frac{\partial s}{\partial x} d y
$$

From this it follows that a minimization of the integral of across track gradients in case of along track uncorrelated striping noise can suppress this kind of noise. However, $\frac{\partial i m}{\partial x}$ is unknown and, hence, an approximation is necessary that can only be derived from $\mathrm{im}_{s}$.

For this, the along track integral of Equation (3) is substituted with the row median med $d_{y}$ and with the across track integral to account for dominating across track gradients and to enable a direct determination of $\underline{s}$ which gives then the following Equation:

$$
\int_{\Omega}\left(\operatorname{med}_{y} \frac{\partial i m-i m s}{\partial x}\right) \otimes 1_{w}(y) d x=\int_{\Omega}\left(\operatorname{med}_{y} \frac{\partial s}{\partial x}\right) \otimes 1_{w}(y) d x=\int_{\Omega} \frac{\partial s}{\partial x} d x=s
$$

The problem to estimate $\frac{\partial i m}{\partial x}$ still remains, but now a gradient based solution is given for $s$, because the term $\left(\operatorname{med}_{y} \frac{\partial s}{\partial x}\right) \otimes 1_{w}(y)$ equals $\frac{\partial s}{\partial x}$. This relies on the principle that scene invariant along track stripes have no along track variation. In this work $\frac{\partial \mathrm{im}}{\partial x}$ is estimated by the difference of $\frac{\partial \mathrm{im}_{s}}{\partial x}$ and its along track smoothed representation that is basically a column high pass filtering result of $\frac{\partial i m_{s}}{\partial x}$. This gives then the following relation:

$$
\frac{\partial i m}{\partial x} \approx \frac{\partial i m_{s}}{\partial x}-\frac{\partial i m_{s}}{\partial x} \oplus f i l_{1}
$$

where $f i l_{1}$ denotes a column filter vector of length 2 and valued with -1 and 1 and $\oplus$ is the convolution operator. This results in an approximation of $\frac{\partial i m}{\partial x}$, because the right hand side of Equation (5) can be considered as an along track high pass filtering result, where mostly real image gradients remain depending on the selection of the elements of the filter. An example is shown in Figure 4. Depending on the selection of the filter and its size the result may vary as shown in Figure 4. Basically, strong smoothing filters tend to suppress image gradient information and should be avoided. In this work a Boxcar filter of size 3 was selected, but other isotropic filters might be also appropriate. 
Figure 4. False colored near infrared image subsets that show the process of the estimation of the across track gradients considering Equation (5) for an HyMAP data set acquired over Potsdam in 2004 (a), that was artificially degraded with uncorrelated across track noise (b), the across track gradient from (a) in (c), the across track gradient from (b) in (d) and the estimation of $\frac{\partial \mathrm{im}}{\partial x}$ from $\mathrm{im}_{s}$ in (e).

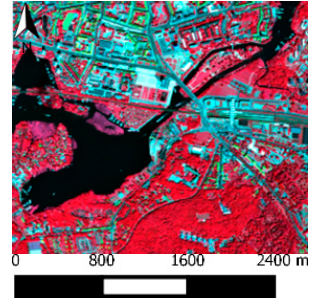

(a)

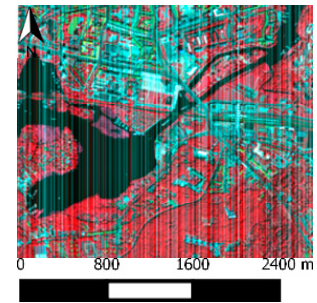

(b)

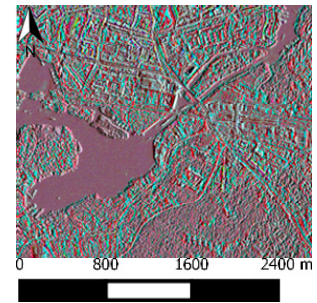

(c)

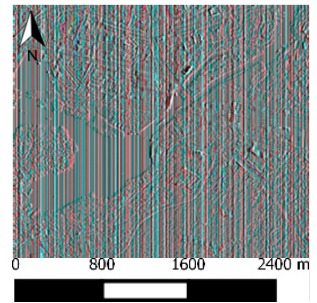

(d)

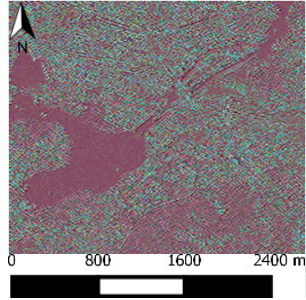

(e)

If both Equations (4) and (5) are combined, then the following Equation (6) relates to the assessment of $s$ :

$$
\int_{\Omega} \operatorname{med}_{y}\left(\frac{\partial i m_{s}}{\partial x} \oplus f i l_{2}\right) \otimes 1_{w}(y) d x \approx s
$$

where $\mathrm{fil}_{2}$ denotes a column Boxcar filter of length 3 that is used to suppress the impact of impulse noise on succeeding stripe noise estimation. To avoid small errors in the assessment of $s$ and to avoid overshooting artifacts (overcorrection) as described in [4,9], the following post-processing steps can be additionally applied. At first, $s$ is reduced by its mean to integrate the zero mean property of thermal Gaussian noise (like dark current variations), $s-\bar{s}$ is then applied on the striped image and the difference of the current mean and the mean before applying $s$ is subtracted from the image. This then gives the following Equation:

$$
i m \approx i m_{s}-s+\bar{s}-\overline{i m_{s}-s+\bar{s}}+\overline{i m_{s}}=i m_{d s 1}
$$

Second, potential overcorrections and other originated uncorrelated striping noise is reduced such as stray light that could be visually perceptible as long wave brightness gradients. This is performed by estimating the column medians, a strong smoothing of those medians and by subtracting difference of the current mean and the mean before applying $\underline{s}$ as in the previous step. This gives then the relation:

$$
i m \approx i m_{d s 1}-\left(\operatorname{med}_{y}\left(i m_{d s 1}\right) \oplus f i l_{3}\right) \otimes 1_{w}-\left(\left(\overline{\left.\operatorname{med}_{y}\left(i m_{d s 1}\right) \oplus f i l_{3}\right) \otimes 1_{w}}\right)+\overline{i m_{s}}=i m_{d s 2}\right.
$$

where $\mathrm{fil}_{3}$ denotes a broad filter that is basically used to reduce approximated long wave trends as suggested in [43]. Here we used the half of the image width or columns. Other filter sizes or filtering approaches may also work such as those described in [44], but were not tested. This might be part of future research. The selected approach is similar to [4,9], but the trends are now subtracted and mean normalized instead of being divided. It follows from this that deviations between true and estimated trends have now a lower influence on the overall destriping performance. Nevertheless, it is recommended to transform each image from at-sensor-radiance to at-sensor-reflectance to avoid drawbacks on detrending caused by varying illumination geometry. Additionally, high non-stationary dark current bias should be reduced beforehand by applying the approach of [10]. 


\subsection{Evaluation Metrics}

\subsubsection{For Artificially Degraded Images (HyMAP)}

All inspected approaches have been evaluated according their performance using the following four metrics to avoid relying on a single performance criterion. The first metric is the Peak-Signal-To-Noise ratio (PSNR) [45]. According to [4] the PSNR is a ratio between band maximum and standard deviation and can be considered as a contrast measurement. The second metric is the local Modified Structural Similarity Index (MSSIM) [17,45,46]. It combines local structure, luminance and contrast metrics and be considered as an overall image (band) quality index. The third metric is the column correlation and the fourth metric is the overall correlation both between ground truth and the destriping result. The two last metrics can be considered as spectral preservation indices. All four performance indicators have been related to available ground truth (the original HyMAP scenes) and, hence, these metrics enable objective evaluations.

\subsubsection{For Striped Real Images (Hyperion/AISA Dual)}

The evaluations of the performance of each considered approach has been differently performed for real images compared to artificially striped images because ground-truth was not available. Hence, PSNR, MSSIM, column and overall correlations have not been applied for the performance evaluation. In addition, relative approaches as proposed in [4] are limited according to their performance evaluation significance.

In this work, three approaches are used for the evaluation of the performance of the destriping results. Those are the subjective visual inspection and two objective indices. The first objective index basically incorporates the property of uncorrelated striping noise to have zero mean that is additionally biased by potential impulse noise. Therefore, the differences between the original image and the destriped images should also have a zero mean of column averages if the striping noise have been exactly estimated. This is biased if high non-stationary dark current noise still impacts the data. To suppress this, the approach of [10] should be applied before any succeeding destriping. However, a potential destriping index may consider this property if it is assumed that remaining striping noise has this property. A related index can be defined as follows:

$$
\begin{gathered}
\operatorname{diff}=\left(\operatorname{im}_{d s 2}(x, y=1)-\operatorname{im}_{s}(x, y=1)\right) \oplus f_{i l}=\tilde{s}(x, y=1) \oplus \mathrm{fil}_{4} \\
A A H P D=\left|\overline{\text { diff }-\operatorname{diff} \oplus f i l_{4}}\right|
\end{gathered}
$$

with $\tilde{s}(x, y=1)$ as the first row of the estimated stripe matrix of different approaches, fil $_{4}$ as boxcar filter of size 3 and AAHPD as Absolute Average of Highpass of Differences. Contrary to a straightforward evaluation of the average of $\tilde{s}(x, y=1)$ this index AAHPD also incorporates remaining low frequency overcorrections through the second filtering step as given in Equation (10). This also enables a more objective evaluation of approaches that include a mean centering of estimated stripe matrix as the proposed approach integrates.

As previously mentioned, AAHPD should be close to zero if the destriping approach has significantly reduced uncorrelated striping noise. In addition to the AAHPD, the correlation of the vectors of the 
integrals of the absolute along track gradients (CIAG) of the original and the destriped image were included for performance evaluation purposes which terms are given in the following as:

$$
\int_{\Omega}\left|\frac{\partial i m_{s}}{\partial y}\right| d y \wedge \int_{\Omega}\left|\frac{\partial i m_{d s 2}}{\partial y}\right| d y
$$

Here, the principle utilized is that stationary along track stripes do not change along track image gradients and, hence, any destriping must preserve them as well. For the sake of simplicity, the median and the $3 \sigma$ standard deviation were used as performance metrics for the correlation vectors consisting of the correlation values for each band.

The visual inspection has been performed using false colored images of bands in which uncorrelated striping noise is visually straightforward perceptible. The interpretation of the results has been additionally ranked as for the other evaluation metrics to avoid relying on a single criterion or imbalanced weighting criteria.

\section{Results and Discussion}

The evaluation of the results of different approaches has been performed using the concepts given in preceding chapters. All performance indicators have been averaged using their respective median that is more robust than the geometric mean if outliers are present, e.g. the destriping results for atmospheric absorptions bands. Additionally, three-sigma probability estimates have been computed to account for the performance variation of different approaches. Both the median and the three-sigma probability estimate of the inspected performance indicator may characterize the performance better than a single statistical criterion. If possible, all indicators were related to ground truth observations (HyMAP). All tested approaches have been applied according to the suggestions of respective authors. Therefore, achieved results may not represent potential results if the parameter selection of parameterized approaches would be optimized for each scenario.

\subsection{For Artificially Degraded Images (HyMAP)}

The averaged results for all approaches, bands and noise scenarios are given in the following Table 1 and will now be analyzed and discussed in more detail. Considering the PSNR as contrast indicator that is exemplarily shown in Figure 5 the proposed approach performs best that is closely followed by the approach of [13] and related to Table 1 more distant by the approach of [10,11] as well as by the approach of [9].

However, the new approach has a significantly lower variation of achieved PSNR and remains stable even in low SNR scenarios as in the bands that are spectrally close to atmospheric absorptions. If the three-sigma probability estimates are additionally considered then the approach of [9] slightly outperforms the approach of [10,11], but this is not valid in low SNR scenarios where stripes contribute more to the overall signal variation than in high SNR scenarios.

If the two best performing approaches, which are related to PSNR - the proposed approach and the approach of [13] - are considered more in detail as exemplarily shown in Supplementary Figure S1, then the approach of [13] slightly outperforms the proposed approach mostly in high SNR scenarios. On 
average, the proposed approach performs best whereas the performance mostly varies close to the 1.4 $\mu \mathrm{m}$ water vapor absorption.

When the MSSIM indicator is considered, which represents an overall image quality index, then a performance ranking according the results is quite similar to those related to the PSNR indicator as exemplarily shown in Figure 5. However, the differences between the best approach - the proposed approach — and the second best — the approach of [13] — are more significant as depicted in Figure 5. Contrary to PSNR, the proposed approach outperforms the second best approach in each band on average. This is also valid for the performance variation (compare Table 1) that is factors smaller for the proposed approach compared to the second best approach of [13].

Table 1. Objectively derived results for simulated images.

\begin{tabular}{ccccccc}
\hline Approach/Indicator & M1 & M2 & M3 & M4 & M5 & M6 \\
\hline Average PSNR [\%] & 99.92 & 97.09 & 97.20 & 83.50 & 99.81 & 85.87 \\
$(3 \sigma)$ & $(0.30)$ & $(11.22)$ & $(14.45)$ & $(19.97)$ & $(4.56)$ & $(27.54)$ \\
\hline Average MSSIM [\%] & 99.58 & 93.40 & 93.43 & 80.57 & 95.68 & 65.94 \\
$(3 \sigma)$ & $(1.43)$ & $(29.99)$ & $(29.92)$ & $(46.47)$ & $(22.79)$ & $(24.07)$ \\
\hline Average Column Correlation[\%] & 99.96 & 91.34 & 86.19 & 78.65 & 93.77 & 95.77 \\
$(3 \sigma)$ & $(0.4)$ & $(11.54)$ & $(8.70)$ & $(15.73)$ & $(6.72)$ & $(7.23)$ \\
\hline Average Spectral Correlation [\%] & 99.93 & 91.35 & 86.03 & 76.39 & 91.97 & 94.37 \\
$(3 \sigma)$ & $(3.32)$ & $(49.06)$ & $(47.76)$ & $(62.19)$ & $(27.51)$ & $(55.55)$ \\
\hline Average [\%] & $\underline{99.85}$ & $\underline{93.29}$ & $\underline{90.71}$ & $\underline{79.78}$ & $\underline{95.31}$ & $\underline{85.49}$ \\
$(3 \sigma)$ & $\underline{(1.36)}$ & $\underline{(25.45)}$ & $\underline{(25.21)}$ & $\underline{(36.09)}$ & $\underline{(15.40)}$ & $\underline{(28.60)}$ \\
\hline
\end{tabular}

M1: Proposed approach; M2: Rogass et al. (2012) [9]; M3: Goodenough et al. (2003) and Datt et al. (2003) [10,11];

M4: Staenz et al. (2002) [12]; M5: Pande-Chhetri and Abd-Elrahman (2011) [13]; M6: Pande-Chhetri and Abd-Elrahman (2013) [14].

Figure 5. Average MSSIM of the two best performing approaches for artificially degraded images.

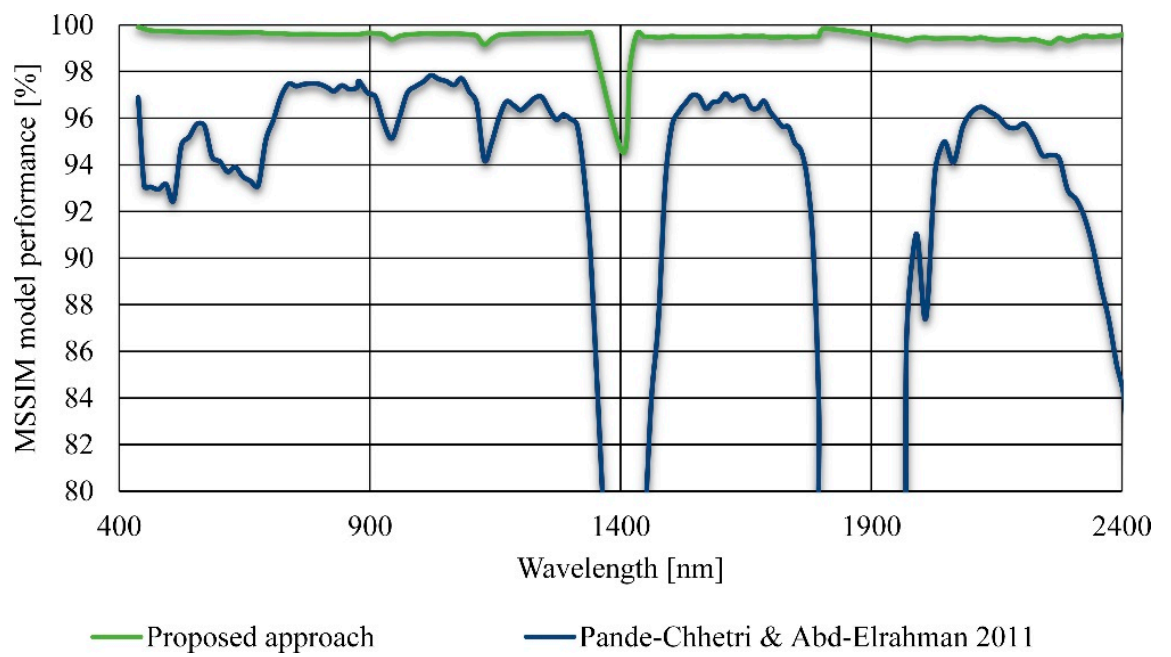

The column correlation index relates the correlation between spatially adjacent image columns and is mostly close to 1 , because the surface cover only slowly varies from column to column. Therefore, any remaining stripes would decrease the correlation coefficient. Consequently, the higher the index the 
more the stripes have been reduced or the more the spectra have been preserved. Here, the proposed approach significantly outperforms the second best approach of [14] that is exemplarily shown in Supplementary Figure S2 that is contrary to the performance ranking for the PSNR and the SSIM indicators. This might be caused by the overall smoothing effect that is derived if the approach of [14] will be applied. Contrary to the column correlation the proposed approach and the approach of [13] perform best that is similar to the PSNR and MSSIM results. Additionally, the performance of the second best approach significantly decreases with increasing noise level as shown in Supplementary Figure S3.

According to all the four performance indicators - PSNR, SSIM, Column and Overall Correlation - the proposed approach performs best. The second best approach is on average the approach of Pande-Chhetri and Abd-Elrahman [13]. If the objectively obtained results were extended by visual inspection, using for example the result subsets that are exemplarily shown in Figure 6, then the approach of Goodenough et al. and Datt et al. [10,11] would be ranked as the second best approach. This is clearly visually perceptible while comparing the "stripiness" of, e.g., the dark colored lake of Figure $6 \mathrm{~d}-\mathrm{g}$.

Integrating all objectively and subjectively obtained results for artificially striped HyMAP images led to a performance ranking that is given in Supplementary Table 4. The proposed approach performs best, second is the image domain approach of Pande-Chhetri and Abd-Elrahman [13] and third are the globally applied methods of Goodenough et al. and Datt et al. [10,11]. If the different destriping results are compared only on a visual basis, then all approaches including the proposed one significantly suppress stripes, but some residual striping is still remaining that may then hinder succeeding analysis, such as a chlorophyll content estimation of the lake in the south west of the subsets as shown in Figure $6 \mathrm{~d}-\mathrm{h}$. If the overall results for simulated images would be quantized to three level of destriping, then the proposed approach and the image domain approach of Pande-Chhetri and Abd-Elrahman [13] would achieve the highest stripe reduction level, the approaches of Goodenough et al. and Datt et al. [10,11] and Rogass et al. [9] a moderate stripe reduction level and the approach of Staenz et al. [12] and the MNF domain approach of Pande-Chhetri and Abd-Elrahman [14] a low stripe reduction level.

Figure 6. Exemplary results as false colored contrast enhanced subsets of one HyMAP scene (a); Potsdam original; figured again for visual comparison) that has been artificially degraded with 5\% noise (b) for (c) the proposed approach, (d) Rogass et al. [9], (e) Goodenough et al. and Datt et al. [10,11], (f) Staenz et al. [12], (g) Pande-Chhetri and Abd-Elrahman ([13] and (h) Pande-Chhetri and Abd-Elrahman [14].

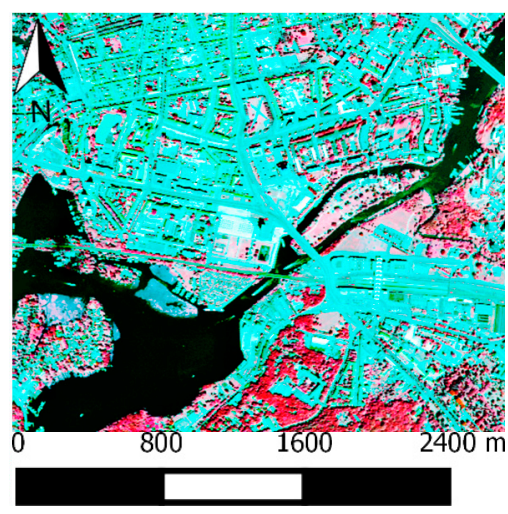

(a)

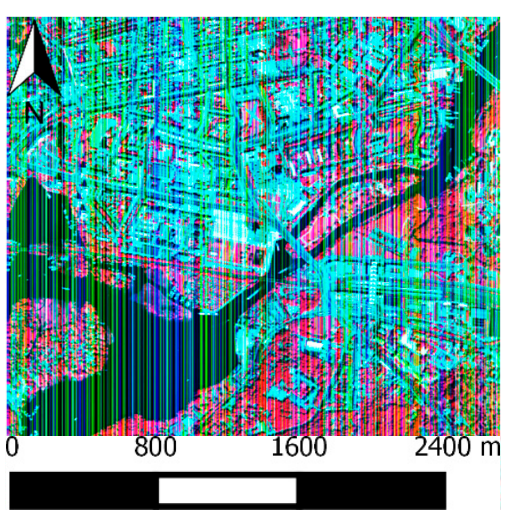

(b) 
Figure 6. Cont.

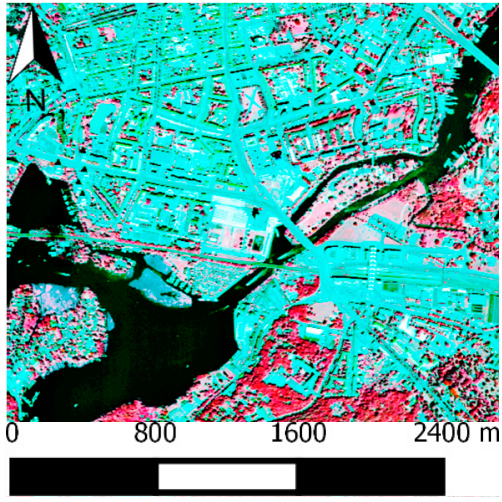

(c)

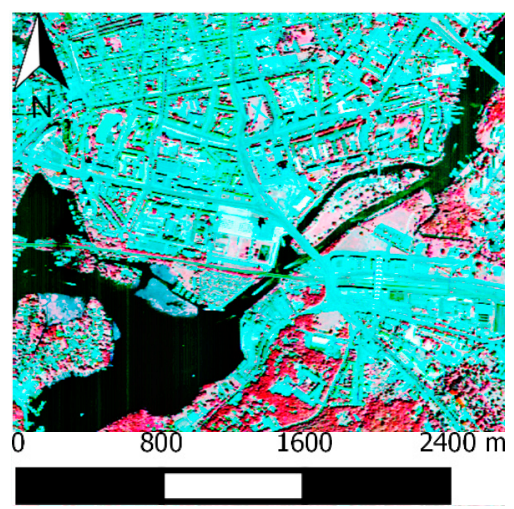

(e)

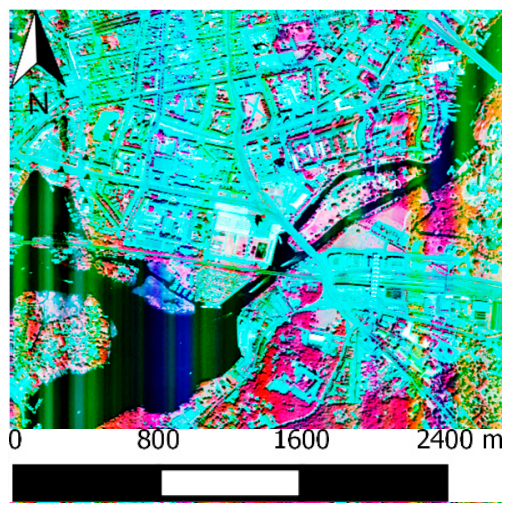

(g)

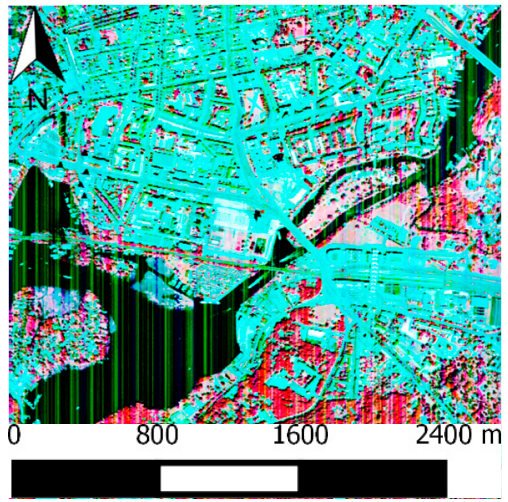

(d)

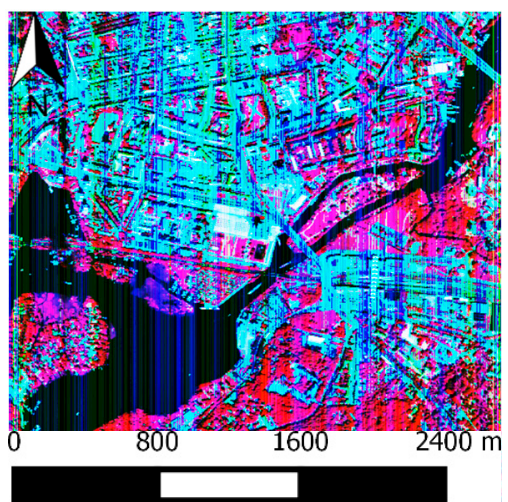

(f)

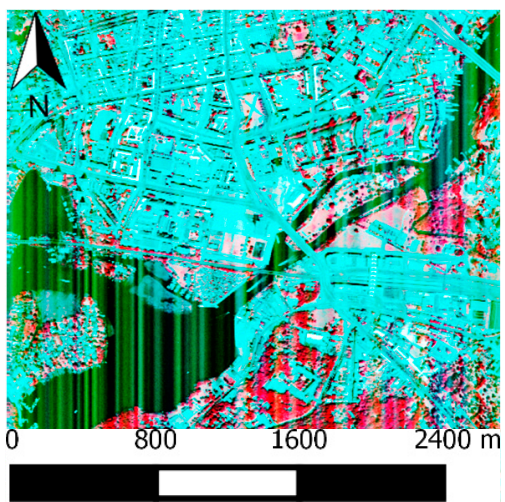

(h)

\subsection{For Real Images (Hyperion/AISA Dual)}

In the following section, the destriping results for originally striped remote sensing images (Hyperion/AISA Dual) are evaluated. Contrary to artificially degraded images, the ground truth was not available that may bias any kind of evaluation. However, the performance evaluation was here also subjectively and objectively conducted. The objective evaluation results are given in Table 2 .

With regard to the results of Table 2, the proposed approach outperforms all other approaches on average. The second best approach is on average the approach of Rogass et al. [9]. The third best approach is on average the image domain approach from Pande-Chhetri and Abd-Elrahman [13]. The differences between the first and the second best approach are significant, and that coincides with the 
evaluation results obtained for simulated images as perceptible in Figure 5 and the Supplementary Figures S1-S3.

Contrary to Rogass et al. [9] the proposed approach mostly achieves a decreased destriping performance in significant low SNR scenarios as exemplarily shown in Supplementary Figure S1. Although the estimation of the overall performance is strongly impacted by the results for low SNR scenarios, it is important to include those results to enable both "worst case" performance evaluations and the incorporation of bands that are "indirectly" useful for, e.g., estimation of columnar water vapor.

Table 2. Objectively derived results for real images.

\begin{tabular}{ccccccc}
\hline Approach/Indicator & M1 & M2 & M3 & M4 & M5 & M6 \\
\hline Median AAHPD/Hyperion [\#] & 0.00004 & 0.00028 & 0.00034 & 0.00084 & 0.00060 & 0.00166 \\
$(3 \sigma)$ & $(0.0029)$ & $(0.0021)$ & $(5.5623)$ & $(0.3825)$ & $(0.0031)$ & $(0.0136)$ \\
\hline Median AAHPD/AISA [\#] & 0.02311 & 0.0634 & 0.18276 & 0.05955 & 0.06011 & 0.09223 \\
$(3 \sigma)$ & $(1.3208)$ & $(1.3666)$ & $(157.7)$ & $(1.3317)$ & $(1.4045)$ & $(1.8410)$ \\
\hline Average AAHPD [\#] & 0.00962 & 0.03144 & 0.04128 & 0.02516 & 0.02733 & 0.031 \\
$(3 \sigma)$ & $(0.6476)$ & $(0.6557)$ & $(59.052)$ & $(0.9894)$ & $(0.6759)$ & $(0.7650)$ \\
\hline Median CIAG/Hyperion [\#] & $\underline{1.0}$ & $\underline{1.0}$ & $\underline{0.88}$ & $\underline{0.97}$ & $\underline{0.51}$ & $\underline{0.38}$ \\
$(3 \sigma)$ & $\underline{(0.21)}$ & $\underline{(0.66)}$ & $\underline{(0.71)}$ & $\underline{(0.84)}$ & $\underline{(0.93)}$ & $\underline{(0.81)}$ \\
\hline Median CIAG/AISA [\#] & $\underline{1.0}$ & $\underline{1.0}$ & $\underline{0.06}$ & $\underline{0.24}$ & $\underline{1.0}$ & $\underline{0.19}$ \\
$(3 \sigma)$ & $(0.25)$ & $\underline{(0.1)}$ & $(0.68)$ & $\underline{(0.63)}$ & $\underline{(0.03)}$ & $\underline{(0.34)}$ \\
\hline Average CIAG [\#] & 1.0 & 1.0 & 0.47 & 0.6 & 0.76 & 0.28 \\
$(3 \sigma)$ & $(0.23)$ & $(0.38)$ & $(0.70)$ & $(0.74)$ & $(0.48)$ & $(0.57)$ \\
\hline
\end{tabular}

M1: Proposed approach; M2: Rogass et al. (2012) [9]; M3: Goodenough et al. (2003) and Datt et al. (2003) [10,11]; M4: Staenz et al. (2003) [12]; M5: Pande-Chhetri and Abd-Elrahman (2011) [13]; M6: Pande-Chhetri and Abd-Elrahman (2013) [14].

Figure 7. Average AAHPD performance of the two best performing approaches for real images.

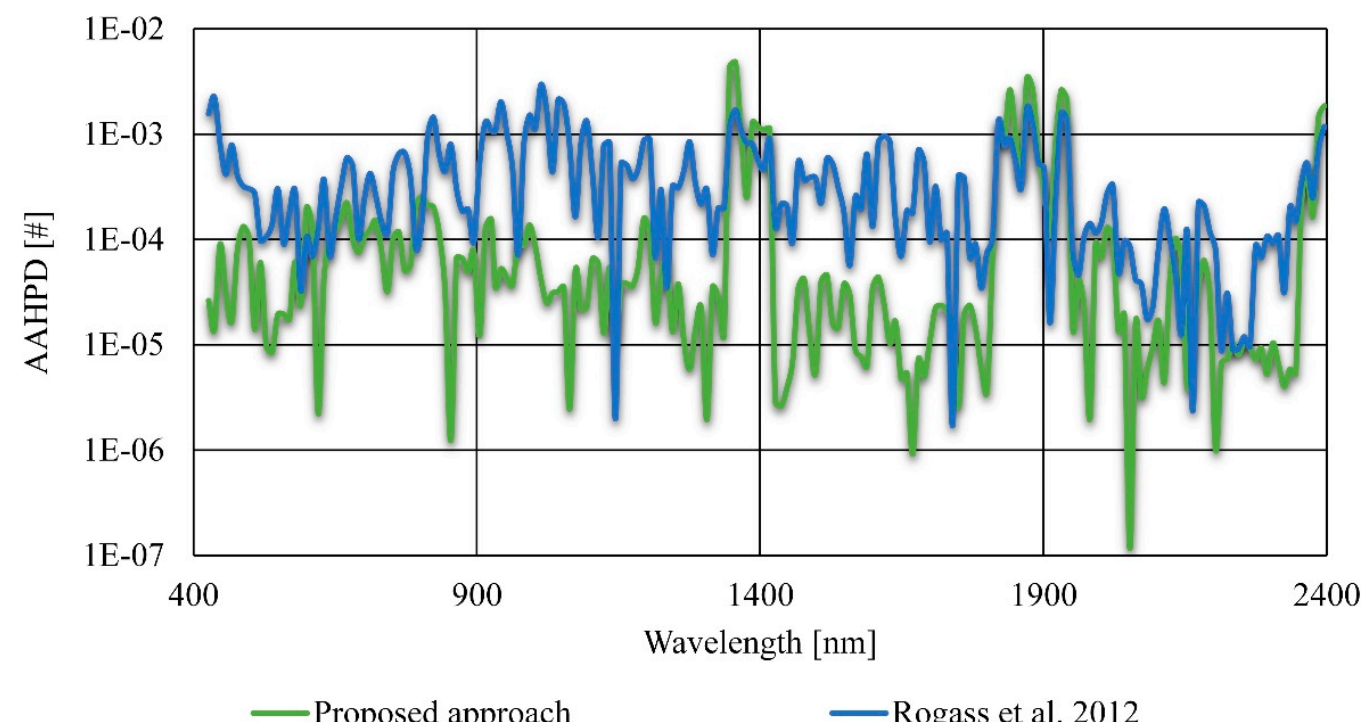

As for simulated images, the subjective evaluation (visual) has been performed because even very fine differences are often more easily perceptible through human vision as through statistical approaches. The succeeding ranking was based on a result evaluation of subsets that are exemplarily shown in Figure 8. 
Figure 8. Subsets of one Hyperion scene (a) as across track spatially stretched false color composites (Red $2304.71 \mathrm{~nm}$, Green $915.23 \mathrm{~nm}$, Blue $447.17 \mathrm{~nm}$ ) of the destriping results of (b) the proposed approach, (c) Rogass et al. [9], (d) Goodenough et al. and Datt et al. [10,11], (e) Staenz et al. [12], (f) Pande-Chhetri and Abd-Elrahman [13] and (g) Pande-Chhetri and Abd-Elrahman [14].

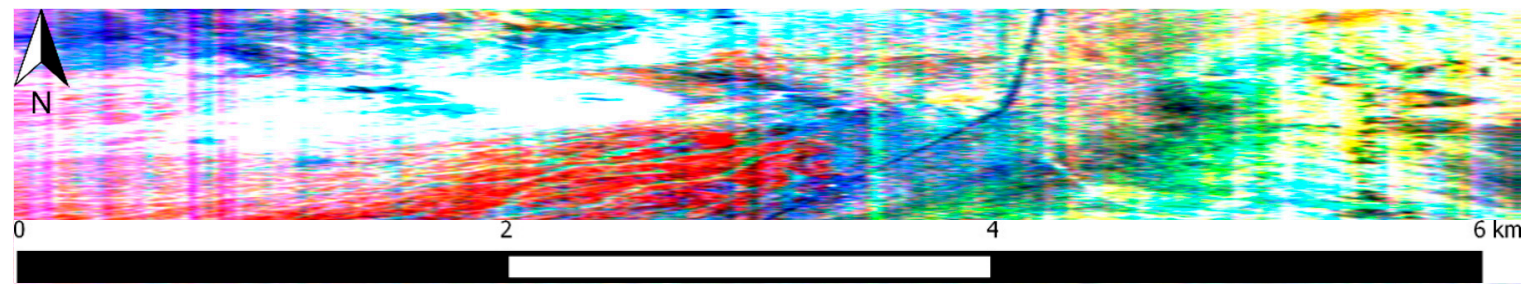

(a)

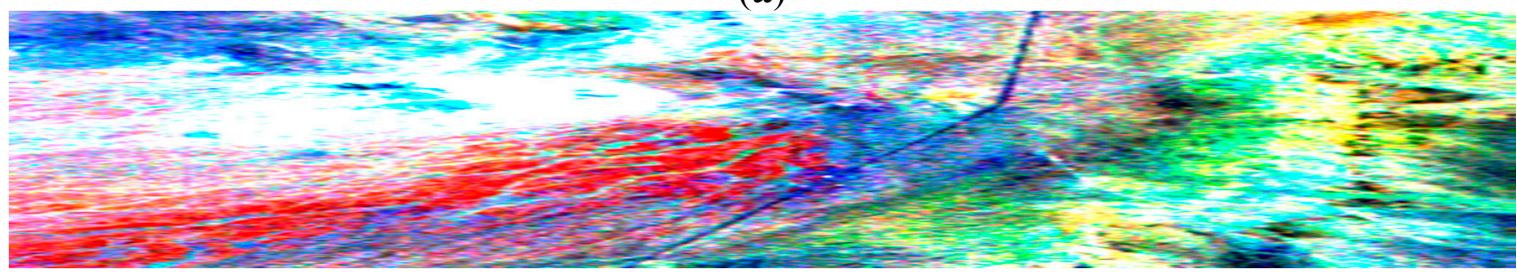

(b)

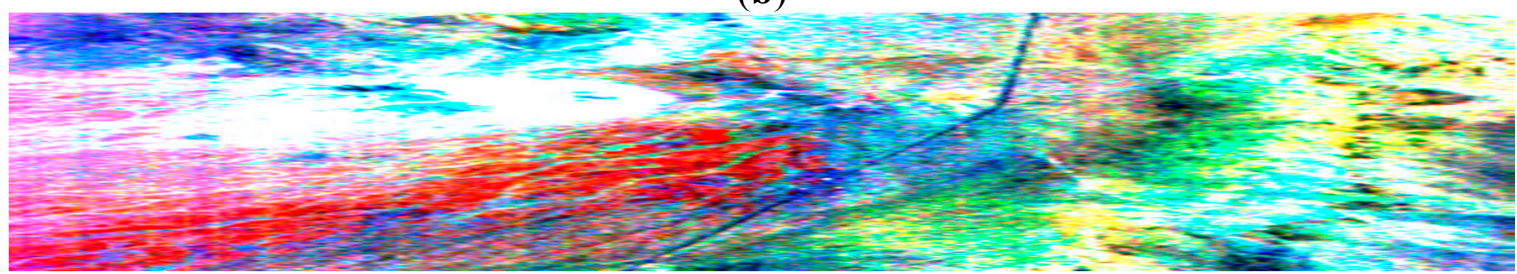

(c)

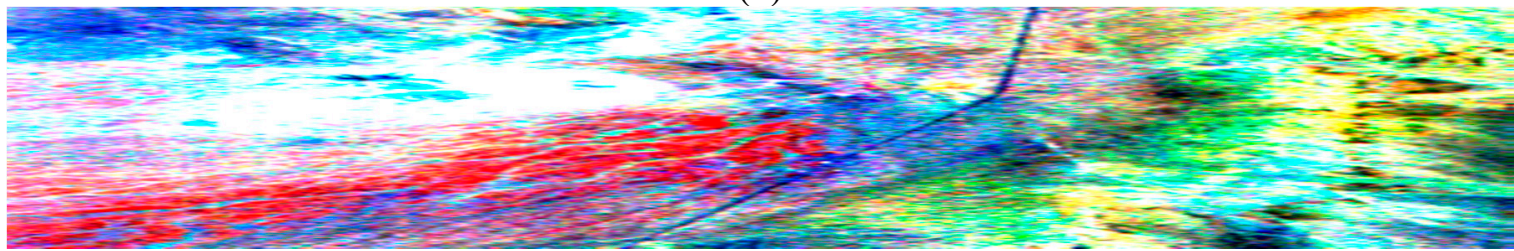

(d)

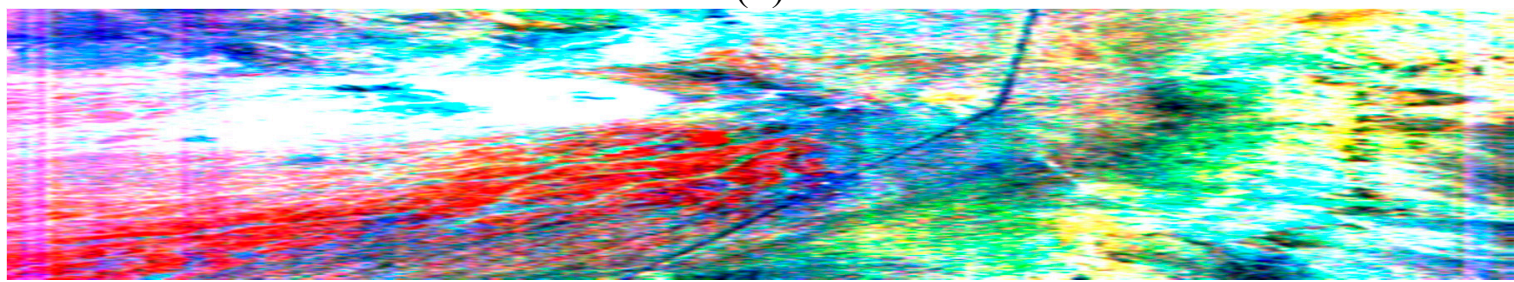

(e)

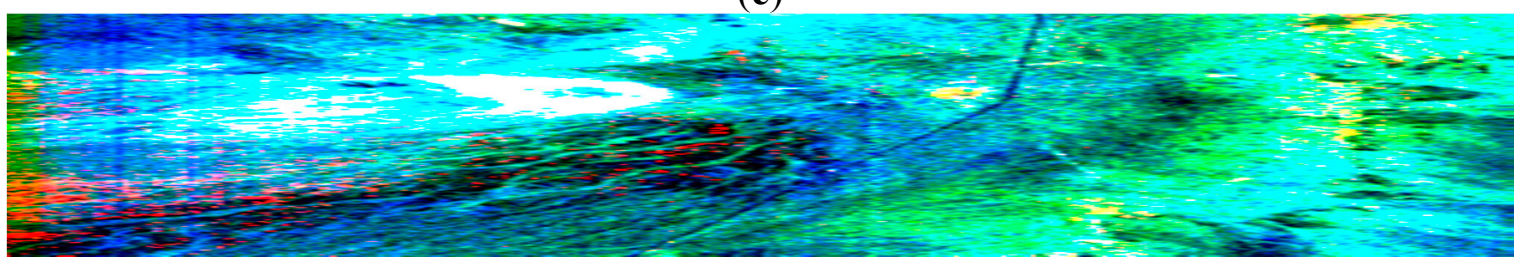

(f)

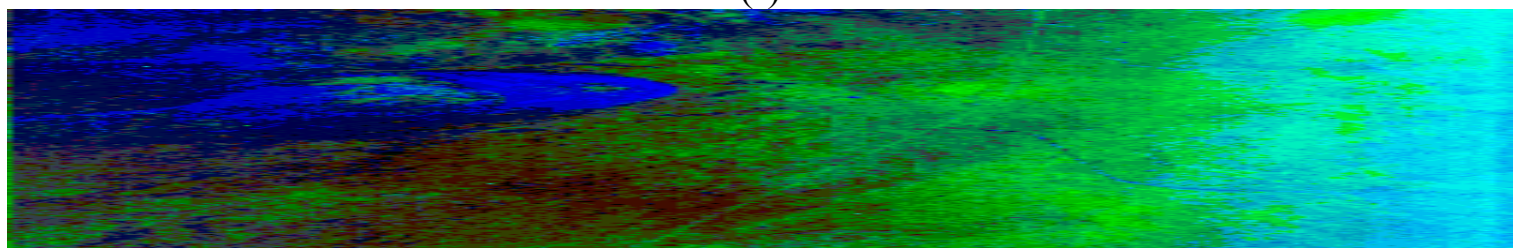

(g) 
The results for the ranking are given in Supplementary Table S5. Based on the visual comparison, both the proposed approach and the approach of Goodenough et al. and Datt et al. [10,11] perform best. This is similar for the overall Hyperion related ranking, whereas the approach of Rogass et al. [9] performs as second best and the approach of Goodenough et al. and Datt et al. $[10,11]$ as third best.

Figure 9. Subsets of one AISA Dual scene (a) as across track spatially stretched false color composites (Red $1574.37 \mathrm{~nm}$, Green $964.39 \mathrm{~nm}$, Blue $730.05 \mathrm{~nm}$ ) of the destriping results of (b) the proposed approach, (c) Rogass et al. [9], (d) Goodenough et al. and Datt et al. [10,11], (e) Staenz et al. [12], (f) Pande-Chhetri and Abd-Elrahman [13] and (g) Pande-Chhetri and Abd-Elrahman [14].

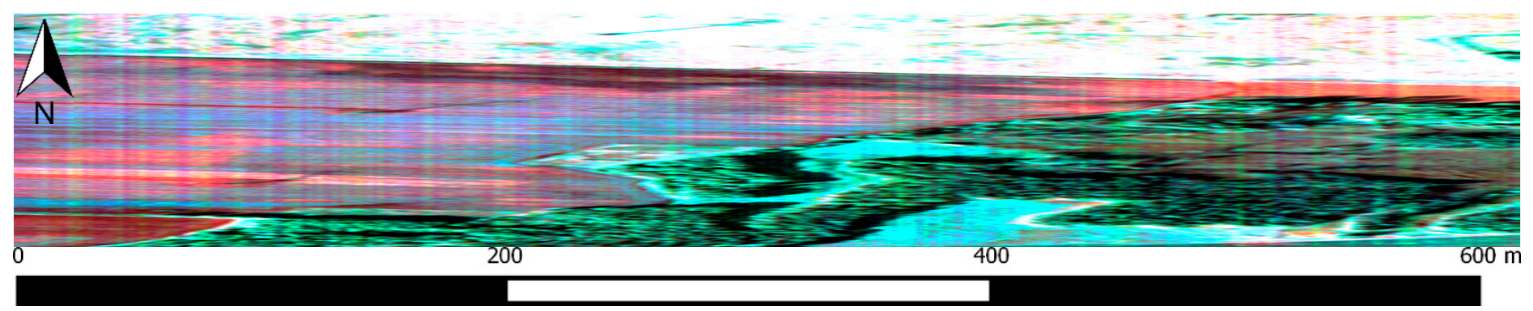

(a)

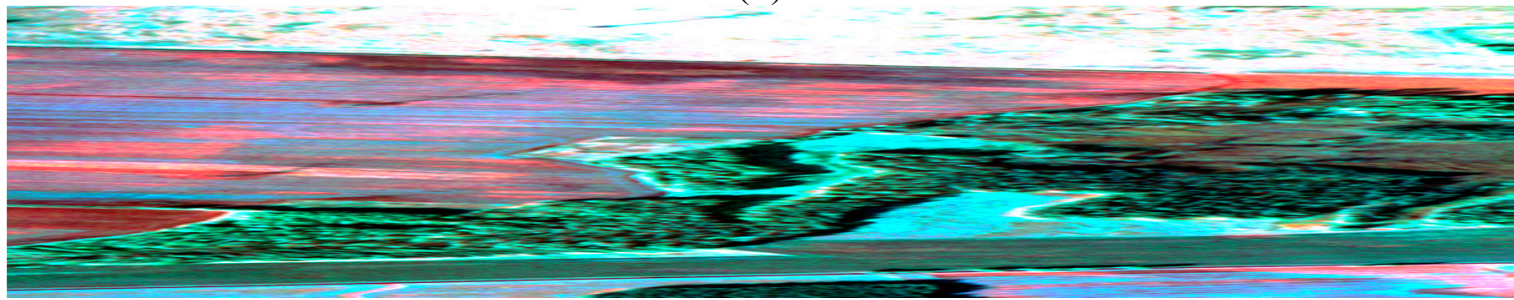

(b)

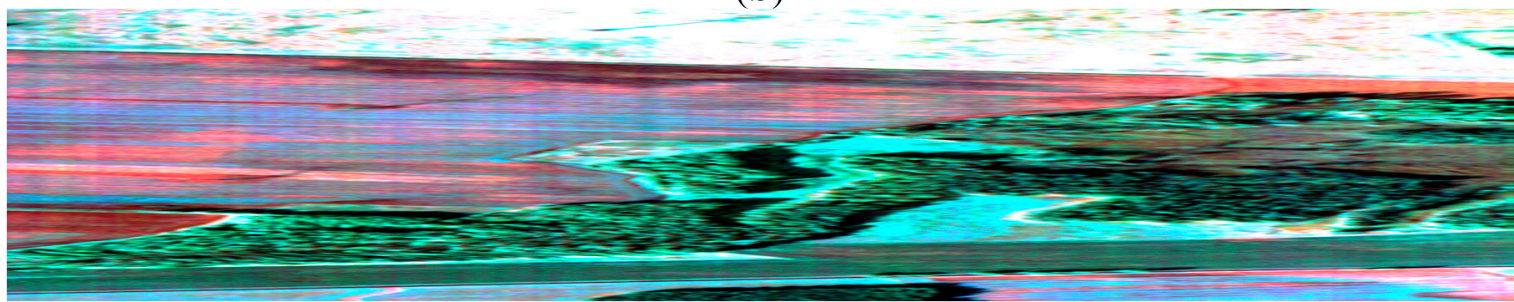

(c)

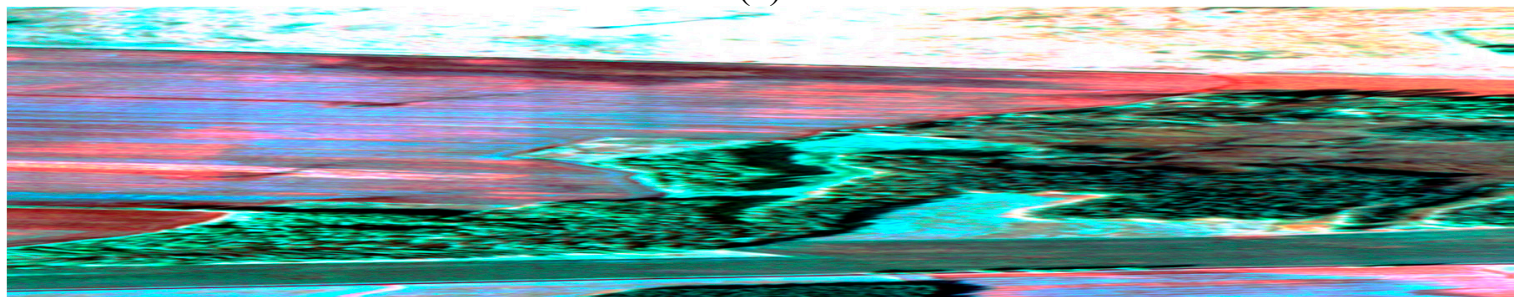

(d)

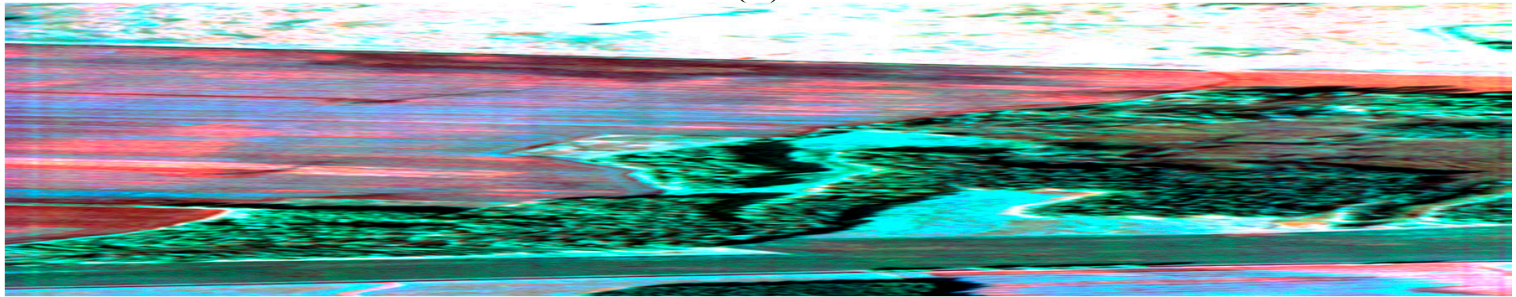

(e) 
Figure 9. Cont.

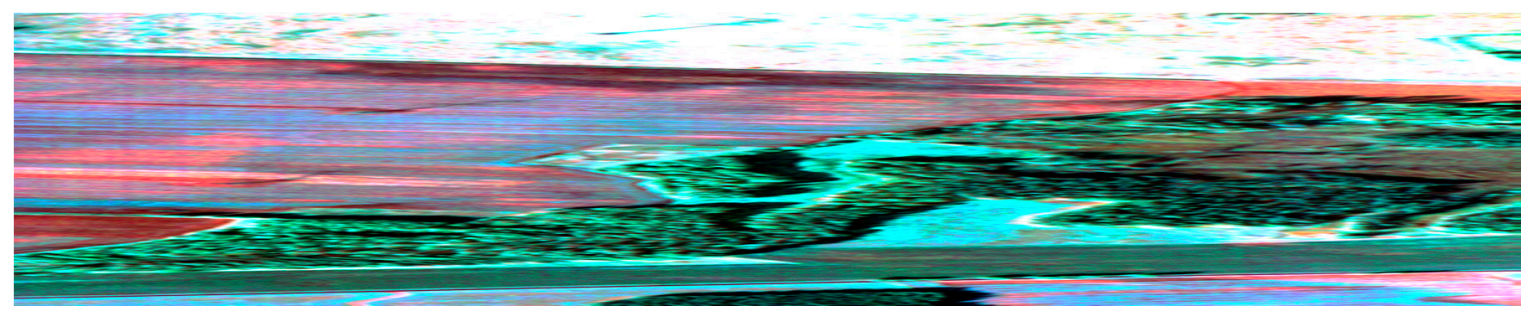

(f)

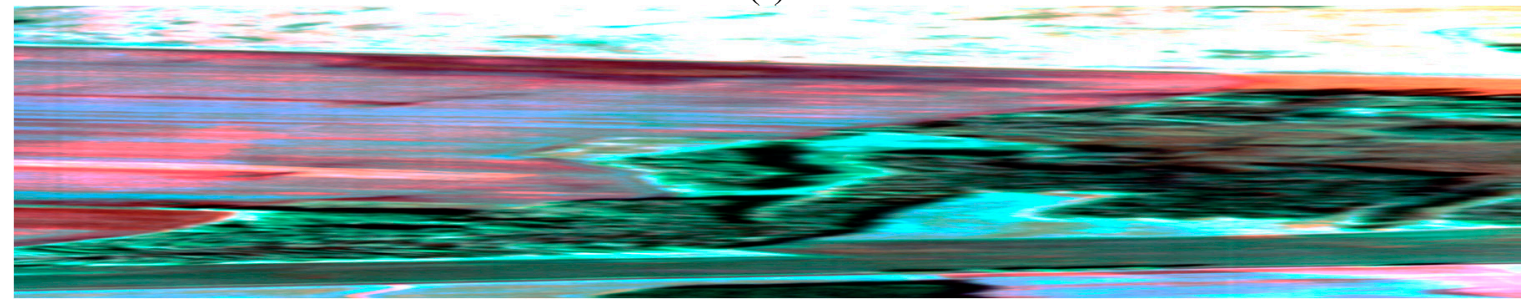

$(\mathbf{g})$

The ranking of Supplementary Table S5 is partly in contrast to the overall objectively obtained results of Supplementary Table S4. It follows from this that different SNR scenarios lead to a variation of individual ranking except for the proposed approach. This assumption is supported while considering the visual based ranking for the individual results based on real striped AISA Dual acquisitions that are exemplarily shown in Figure 9. There the proposed approach and the approach of Goodenough et al. and Datt et al. [10,11] perform best. Contrary to the visually obtained results for Hyperion (compare Supplementary Table S5) the approach of Pande-Chhetri and Abd-Elrahman [14] performs as second best and, hence, outperforms the approach of Pande-Chhetri and Abd-Elrahman [13]. This might be again caused by sensor related SNR differences (impact of strong smoothing).

As for Hyperion objectively obtained results for destriping AISA Dual images are complemented with the evaluation results based on visual perceptions and summarized in Supplementary Table S6. There, the proposed approach outperforms again all other tested approaches for both scenarios-the statistical and the visual evaluation except for the $3 \sigma$ criterion of the CIAG metric. Contrary to the results for Hyperion the approach of Pande-Chhetri and Abd-Elrahman [13] performs as second best and the approach of Staenz et al. [12] as third best.

\subsection{For Artificially Degraded and Real Images}

If all individual evaluation results for destriping are commonly considered as in Table 3 , then the proposed approach clearly outperforms all other approaches. The second best approach is the approach from Pande-Chhetri and Abd-Elrahman [13] and the third best approach is from Rogass et al. [9]. Both perform similarly, although the approach of Goodenough et al. and Datt et al. [10,11] delivered visually better results. If the subjective evaluation using visual perception will be excluded, then the second and the third best approach switch their ranks. 
Table 3. Overall performance ranking for real AISA Dual and Hyperion images.

\begin{tabular}{ccccccc}
\hline Approach/Indicator & M1 & M2 & M3 & M4 & M5 & M6 \\
\hline PSNR [\#] & 1 & 4 & 3 & 6 & 2 & 5 \\
SSIM [\#] & 1 & 4 & 3 & 5 & 2 & 6 \\
Column Correlation [\#] & 1 & 4 & 5 & 6 & 3 & 2 \\
Overall Correlation [\#] & 1 & 4 & 5 & 6 & 3 & 2 \\
Visual comparison HyMAP [\#] & 1 & 3 & 2 & 6 & 4 & 5 \\
Median AAHPD/Hyperion [\#] & 1 & 2 & 3 & 5 & 4 & 6 \\
Median AAHPD/AISA [\#] & 1 & 4 & 6 & 2 & 3 & 5 \\
Median CIAG/Hyperion [\#] & 1 & 2 & 4 & 3 & 5 & 6 \\
Median CIAG/AISA [\#] & 3 & 2 & 6 & 4 & 1 & 5 \\
Visual comparison Hyperion [\#] & 1 & 3 & 1 & 5 & 2 & 4 \\
Visual comparison AISA [\#] & 1 & 5 & 1 & 3 & 4 & 2 \\
Average [\#] & $\underline{1(1)}$ & $\underline{3(2)}$ & $\underline{4(4)}$ & $\underline{6(5)}$ & $\underline{2(3)}$ & $\underline{5(5)}$ \\
$(\Sigma$ all, $\Sigma$ objectively only) & $\underline{(13,10)}$ & $\underline{(39,35)}$ & $\underline{(51,37)}$ & $\underline{(33,23)}$ & $\underline{(48,37)}$ \\
\hline
\end{tabular}

M1: Proposed approach; M2: Rogass et al. (2012) [9]; M3: Goodenough et al. (2003) and Datt et al. (2003) [10,11];

M4: Staenz et al. (2002) [12]; M5: Pande-Chhetri and Abd-Elrahman (2011) [13]; M6: Pande-Chhetri and

Abd-Elrahman (2013) [14]

\section{Conclusions}

In this work, a new approach for the reduction of signal independent radiometric miscalibration has been proposed. It achieves an accuracy of about $99.8 \%$ for selected artificially degraded image samples. The visual inspection as well as the new defined image quality index for successive destriping indicate that the proposed approach also outperforms all other inspected approaches for real hyperspectral pushbroom acquisitions, such as those from AISA or Hyperion. Because this approach significantly outperforms the latest approach [9] of the ROME framework, it can be expected that destriping of data acquisitions from APEX, ASTER and CHRIS/Proba will be also improved in comparison with the approach described in [9], but this was not tested. These findings are based on the individual results and the overall ranking of the destriping results for all 1536 individually degraded image samples (3 HyMAP scenes, 128 bands and the 4 noise scenarios) and four real image samples. Its high processing speed, the complete vectorization and the striping quality indicator enable an integration into processing chains such as ROME. Compared to the approaches presented by [4,9] this approach is much faster and easier to implement. Integrated filter components are fixed and, therefore, this approach can be considered as parameter free that tend to represent a close approximation of an analytical solution. To avoid drawbacks for any radiometry related destriping approach, it is necessary to reduce acquisition geometry related albedo variations. It follows from this that at-sensor reflectance is recommended as processing level for destriping. It is assumed that other uncorrelated stripes can be also suppressed by the proposed approach if the striping direction is correctly considered in the proposed equations. However, in future work more benchmark tests of more approaches [16-36] are necessary to determine the current best performing approach that should be then integrated into ROME. 


\section{Acknowledgments}

This work was funded by the German Federal Ministry of Economics and Technology (BMWI 50EE1012/EnMAP) within the framework of EnMAP (Environmental Mapping and Analysis Program). We thank the U.S. NASA's Land Processes Distributed Active Archive Center (LP DAAC) for providing image samples. We are grateful to anonymous reviewers and the editors for their constructive and insightful comments that helped to improve the quality of the manuscript.

\section{Author Contributions}

Christian Mielke and Daniel Scheffler contributed the destriping results for the approaches of Goodenough et al. and Datt et al.[10,11], Staenz et al.[12], Pande-Chhetri and Abd-Elrahman [13] and Pande-Chhetri and Abd-Elrahman [14]. Nina K. Boesche, Christin Lubitz and Andreas Eisele provided the visual interpretation of the results, the selection of indicative bands and the metrics for performance evaluations. Maximilian Brell and Karl Segl generalized the mathematical framework of the proposed approach. Daniel Spengler and Angela Lausch carried out the AISA (Airborne Imaging Spectrometer for Applications) acquisitions and performed the pre-processing of the AISA data sets to provide at-sensor radiance for testing. Luis Guanter conducted the atmospheric correction of the HyMAP acquisitions to retrieve at-surface reflectance.

\section{Conflicts of Interest}

The authors declare no conflict of interest.

\section{References}

1. Cocks, T.; Jenssen, R.; Stewart, A.; Wilson, I.; Shields, T. The HyMap ${ }^{\mathrm{TM}}$ Airborne Hyperspectral Sensor: The System, Calibration and Performance. Available online: http://www.neodc.rl.ac.uk/ docs/Hymap_specs.pdf (accessed on 5 September 2014).

2. Pearlman, J.; Carman, S.; Segal, C.; Jarecke, P.; Clancy, P.; Browne, W. Overview of the Hyperion Imaging Spectrometer for the NASA EO-1 mission. In Proceedings of IEEE 2001 International Geoscience and Remote Sensing Symposium, Sydney, Australia, 9-13 July 2001; pp. 3036-3038.

3. Specim. Available online: http://www.specim.fi/index.php/products/airborne/aisadual (accessed on 17 February 2014).

4. Rogass, C.; Spengler, D.; Bochow, M.; Segl, K.; Lausch, A.; Doktor, D.; Roessner, S.; Behling, R.; Wetzel, H.U.; Kaufmann, H. Reduction of radiometric miscalibration-Applications to pushbroom sensors. Sensors 2011, 11, 6370-6395.

5. Oppelt, N.; Mauser, W. The airborne visible/infrared imaging spectrometer AVIS: Design, characterization and calibration. Sensors 2007, 7, 1934-1953.

6. Janesick, J.R. DN to [lambda]; SPIE Press: Bellingham, WA, USA, 2007.

7. Tian, H. Noise Analysis in CMOS Image Sensors. Available online: http://www-isl.stanford.edu/ abbas/group/papers_and_pub/hui_thesis.pdf (accessed on 5 September 2014)

8. Holst, G.C. CCD Arrays, Cameras and Displays; JCD Publishing: Winter Park, FL, USA, 1996. 
9. Rogass, C.; Spengler, D.; Bochow, M.; Segl, K.; Lausch, A.; Doktor, D.; Roessner, S.; Behling, R.; Wetzel, H.U.; Urata, K.; Hueni, A.; Kaufmann, H. A Contribution to the Reduction of Radiometric Miscalibration of Pushbroom Sensors; INTECH Open Access Publisher: Rijeka, Croatia, 2012.

10. Goodenough, D.G.; Dyk, A.; Niemann, K.O.; Pearlman, J.S.; Chen, H.; Han, T.; Murdoch, M.; West, C. Processing Hyperion and ALI for forest classification. IEEE Trans. Geosci. Remote Sens. 2003, 41, 1321-1331.

11. Datt, B.; McVicar, T.R.; Van Niel, T.G.; Jupp, D.L.B.; Pearlman, J.S. Preprocessing EO-1 Hyperion hyperspectral data to support the application of agricultural indexes. IEEE Trans. Geosci. Remote Sens. 2003, 41, 1246-1259.

12. Staenz, K.; Neville, R.A.; Clavette, S.; Landry, R.; White, H.P.; Hitchcock, R. Retrieval of Surface Reflectance from Hyperion Radiance Data. Available online: http://ieeexplore.iee.org/ $\mathrm{xpl} /$ login.jsp?tp=\&arnumber=1026135\&url=http\%3A\%2F\%2Fieeexplore.iee.org\%2Fxpls\%2Fab s_all.jsp\%3Farnumber\%3D1026135 (accessed on 5 September 2014).

13. Pande-Chhetri, R.; Abd-Elrahman, A. De-striping hyperspectral imagery using wavelet transform and adaptive frequency domain filtering. ISPRS J. Photogramm. Remote Sens. 2011, 66, 620-636.

14. Pande-Chhetri, R.; Abd-Elrahman, A. Filtering high-resolution hyperspectral imagery in a maximum noise fraction transform domain using wavelet-based de-striping. Int. J. Remote Sens. 2013, 34, 2216-2235.

15. Scheffler, D.; Karrasch, P. Destriping of hyperspectral image data: An evaluation of different algorithms using EO-1 Hyperion data. J. Appl. Remote Sens. 2014, 8, doi:10.1117/1.JRS.8.083645.

16. Oliveira, P.; Gomes, L. Interpolation of signals with missing data using Principal Component Analysis. Multidimens. Syst. Signal. Process. 2010, 21, 25-43.

17. Tsai, F.; Chen, W.W. Striping noise detection and correction of remote sensing images. IEEE Trans. Geosci. Remote Sens. 2008, 46, 4122-4131.

18. Cavalli, R.M.; Fusilli, L.; Pascucci, S.; Pignatti, S.; Santini, F. Hyperspectral sensor data capability for retrieving complex urban land cover in comparison with multispectral data: Venice city case study (Italy). Sensors 2008, 8, 3299-3320.

19. Liu, B.; Zhang, L.; Zhang, X.; Zhang, B.; Tong, Q. Simulation of EO-1 Hyperion data from ALI multispectral data based on the spectral reconstruction approach. Sensors 2009, 9, 3090-3108.

20. Garcia, J.; Moreno, J. Removal of Noises in CHRIS/Proba Images: Application to the SPARC Campaign Data. Available online: http://earth.esa.int/workshops/chris_proba_04/papers/ 9_GARCIA.pdf (accessed on 5 September 2014).

21. Shen, H.F.; Ai, T.H.; Li, P.X. Destriping and Inpainting of Remote Sensing Images Using Maximum A-Posteriori Method. Available online: http://www.isprs.org/proceedings/XXXVII/congress/ 1_pdf/11.pdf (accessed on 5 September 2014).

22. Simpson, J.J.; Gobat, J.I.; Frouin, R. Improved destriping of GOES images using finite impulse response filters. Remote Sens. Environ. 1995, 52, 15-35.

23. Simpson, J.J.; Stitt, J.R.; Leath, D.M. Improved finite impulse response filters for enhanced destriping of geostationary satellite data. Remote Sens. Environ. 1998, 66, 235-249.

24. Bouali, M.; Ladjal, S. Toward optimal destriping of MODIS data using a unidirectional variational model. IEEE Trans. Geosci. Remote Sens. 2011, 49, 2924-2935. 
25. Carfantan, H.; Idier, J. Statistical linear destriping of satellite-based pushbroom-type images. IEEE Trans. Geosci. Remote Sens. 2010, 48, 1860-1871.

26. Gomez-Chova, L.; Alonso, L.; Guanter, L.; Camps-Valls, G.; Calpe, J.; Moreno, J. Correction of systematic spatial noise in push-broom hyperspectral sensors: Application to CHRIS/PROBA images. Appl. Opt. 2008, 47, F46-F60.

27. Tendero, Y.; Gilles, J. ADMIRE: A locally adaptive single-image, non-uniformity correction and denoising algorithm: Application to uncooled IR camera. Proc. SPIE 2012, 8353, doi:10.1117/12.912966.

28. Shen, H.; Zhang, L. A map-based algorithm for destriping and inpainting of remotely sensed images. IEEE Trans. Geosci. Remote Sens. 2009, 47, 1492-1502.

29. Shen, H.; Liu, Y.; Ai, T.; Wang, Y.; Wu, B. Universal reconstruction method for radiometric quality improvement of remote sensing images. Int. J. Appl. Earth Obs. Geoinf. 2010, 12, 278-286.

30. Yuan, Q.; Zhang, L.; Shen, H. Hyperspectral image denoising employing a spectral-spatial adaptive total variation model. IEEE Trans. Geosci. Remote Sens. 2012, 50, 3660-3677.

31. Bouali, M. A simple and robust destriping algorithm for imaging spectrometers: Application to MODIS data. In Proceedings of ASPRS 2010 Annual Conference, San Diego, CA, USA, 26-30 April 2010; pp. 84-93.

32. Shen, H.; Jiang, W.; Zhang, H.; Zhang, L. A piece-wise approach to removing the nonlinear and irregular stripes in MODIS data. Int. J. Remote Sens. 2013, 35, 44-53.

33. Chen, J.; Shao, Y.; Guo, H.; Wang, W.; Zhu, B. Destriping CMODIS data by power filtering. IEEE Trans. Geosci. Remote Sens. 2003, 41, 2119-2124.

34. Corsini, G.; Diani, M.; Walzel, T. Striping removal in MOS-B data. IEEE Trans. Geosci. Remote Sens. 2000, 38, 1439-1446.

35. Di Bisceglie, M.; Episcopo, R.; Galdi, C.; Ullo, S.L. Destriping MODIS data using overlapping field-of-view method. IEEE Trans. Geosci. Remote Sens. 2009, 47, 637-651.

36. Rakwatin, P.; Takeuchi, W.; Yasuoka, Y. Stripe noise reduction in MODIS data by combining histogram matching with facet filter. IEEE Trans. Geosci. Remote Sens. 2007, 45, 1844-1856.

37. Sang, B.; Schubert, J.; Kaiser, S.; Mogulsky, V.; Neumann, C.; Forster, K.; Hofer, S.; Stuffler, T.; Kaufmann, H.; Muller, A. The EnMAP hyperspectral imaging spectrometer: Instrument concept, calibration and technologies. Imaging Spectrom. 2008, 13, 708605-708615.

38. Hofer, S.; Kaufmann, H.; Stuffler, T.; Penné, B.; Schreier, G.; Müller, A.; Eckardt, A.; Bach, H.; Benz, U.; Haydn, R. EnMAP hyperspectral imager: An advanced optical payload for future applications in Earth observation programs. Proc. SPIE 2006, 6366, doi:10.1117/12.690608.

39. Richter, R.; Schlapfer, D. Geo-atmospheric processing of airborne imaging spectrometry data. Part II: Atmospheric/topographic correction. Int. J. Remote Sens. 2002, 23, 2631-2649.

40. Pearlman, J.S.; Barry, P.S.; Segal, C.C.; Shepanski, J.; Beiso, D.; Carman, S.L. Hyperion, a space-based imaging spectrometer. IEEE Trans. Geosci. Remote Sens. 2003, 41, 1160-1173.

41. Box, G.; Muller, M.E. A note on the generation of random normal deviates. Ann. Math. Stat. 1958, 29, 610-611.

42. Green, A.A.; Berman, M.; Switzer, P.; Craig, M.D. A transformation for ordering multispectral data in terms of image quality with implications for noise removal. IEEE Trans. Geosci. Remote Sens. 1988, 26, 65-74. 
43. Bovik, A.C. Handbook of Image and Video Processing; Academic Press: San Diego, CA, USA, 2000.

44. Arce, G.R. Nonlinear Signal Processing: A Statistical Approach; Wiley-Interscience: Hoboken, NJ, USA, 2004.

45. Wang, Z.; Bovik, A.C. Mean squared error: Love it or leave it? A new look at signal fidelity measures. IEEE Signal. Process. Mag. 2009, 26, 98-117.

46. Wang, Z.; Bovik, A.C.; Sheikh, H.R.; Simoncelli, E.P. Image quality assessment: From error visibility to structural similarity. IEEE Trans. Image Process. 2004, 13, 600-612.

(C) 2014 by the authors; licensee MDPI, Basel, Switzerland. This article is an open access article distributed under the terms and conditions of the Creative Commons Attribution license (http://creativecommons.org/licenses/by/4.0/). 